\title{
NGC 1365: A low column density state unveiling a low ionization disk wind.
}

\author{
V. Braito ${ }^{1,2}$, J. N. Reeves ${ }^{3,4}$,J. Gofford ${ }^{3,4}$, E. Nardini ${ }^{3}$, D. Porquet $^{5}$, G. Risaliti 6,7 \\ ${ }^{1}$ INAF - Osservatorio Astronomico di Brera, Via Bianchi 46 I-23807 Merate (LC), Italy; \\ valentina.braito@brera.inaf.it \\ ${ }^{2}$ ASDC-ASI, Via del Politecnico, 00133 Roma, Italy \\ ${ }^{3}$ Astrophysics Group, School of Physical and Geographical Sciences, Keele University, \\ Keele, Staffordshire ST5 5BG, UK \\ ${ }^{4}$ Department of Physics, University of Maryland, Baltimore County, Baltimore, MD 21250, \\ $U S A$ \\ ${ }^{5}$ Observatoire Astronomique de Strasbourg, CNRS, UMR 7550, 11 rue de l'Université, \\ 67000 Strasbourg, France \\ ${ }^{6}$ INAF - Osservatorio Astrofisico di Arcetri, Largo E. Fermi 5, 50125 Firenze, Italy \\ ${ }^{7}$ Harvard - Smithsonian Center for Astrophysics, 60 Garden Street, Cambridge, MA 02138, \\ USA
}

\begin{abstract}
We present the time-resolved spectral analysis of the $X M M-N e w t o n$ data of NGC 1365 collected during one XMM-Newton observation, which caught this "changing-look" AGN in a high flux state characterized also by a low column density $\left(N_{\mathrm{H}} \sim 10^{22} \mathrm{~cm}^{-2}\right)$ of the X-ray absorber. During this observation the low energy photoelectric cut-off is at about $\sim 1 \mathrm{keV}$ and the primary continuum can be investigated with the XMM-Newton-RGS data, which show strong spectral variability that can be explained as a variable low $N_{\mathrm{H}}$, which decreased from $N_{\mathrm{H}} \sim$ $10^{23} \mathrm{~cm}^{-2}$ to $10^{22} \mathrm{~cm}^{-2}$ in a $100 \mathrm{ks}$ time-scale. The spectral analysis of the last segment of the observation revealed the presence of several absorption features that can be associated with an ionized $\left(\log \xi \sim 2 \mathrm{erg} \mathrm{cm} \mathrm{s}^{-1}\right)$ outflowing wind $\left(v_{\text {out }} \sim 2000 \mathrm{~km} \mathrm{~s}^{-1}\right)$. We detected for the first time a possible P-Cygni profile of the Mg XII Ly $\alpha$ line associated with this mildly ionized absorber indicative of a wide angle outflowing wind. We suggest that this wind is a low ionization zone of the highly ionized wind present in NGC 1365, which is responsible for the iron $\mathrm{K}$ absorption lines and is located within the variable $\mathrm{X}$-ray absorber. At the end of the observation, we detected a strong absorption line at $E \sim 0.76 \mathrm{keV}$ most likely associated with a lower ionization zone of the absorber $(\log \xi \sim 0.2 \mathrm{erg} \mathrm{cm}$ $\mathrm{s}^{-1}, N_{\mathrm{H}} \sim 10^{22} \mathrm{~cm}^{-2}$ ), which suggests that the variable absorber in NGC 1365 could be a low ionization zone of the disk wind.
\end{abstract}


Subject headings: galaxies: individual: NGC 1365 - X-rays: galaxies — galaxies: active

\section{Introduction}

In the last decade several X-ray observations of Seyfert galaxies unveiled a significant variability of the column density $\left(N_{\mathrm{H}}\right)$ or covering fraction of the X-ray absorbers (Risaliti et al. 2002), which highlighted the complexity of the circum-nuclear medium of Active Galactic Nuclei (AGN). The emerging picture is that the X-ray absorber of the widely accepted Unified Model of AGN (Antonucci 1993) is not a single and homogenous structure located at a pc-scale distance from the central source, but most likely multiple absorbers co-exist. These absorbers could be located at different scales at a pc-scale distance from the central nucleus like the putative torus as well as much closer in (i. e. at few tens of gravitational radii) and they could be in part inhomogeneous (Risaliti et al. 2002; Elvis et al. 2004). The advent of the modern X-ray observatory also unveiled that photo-ionized or warm absorbers are common as they are observed in at least $50 \%$ of the unobscured AGN (Reynolds 1997; Porquet et al. 2004; Crenshaw et al. 2003; Blustin et al. 2005). The warm absorbers give rise to several narrow absorption features (from several elements over a wide range of ionisation parameters), which when observed at high spectral resolution are generally blue-shifted by a few hundred $\mathrm{km} \mathrm{s}^{-1}$ up to few thousand $\mathrm{km} \mathrm{s}^{-1}$, implying the presence of outflowing winds. Recently, systematic studies of the X-ray spectra of bright nearby AGN unveiled that blue-shifted absorption features due to FexxV and Fe XXVI are present in at least $40 \%$ of the radio-quiet AGN (Tombesi et al. 2010a; Tombesi et al. 2011, 2012; Patrick et al. 2012; Gofford et al. 2013) and also in a sample of local Broad Line Radio Galaxies (Tombesi et al. 2010b, 2014). In comparison to the soft warm absorbers these winds are characterized by high column densities $\left(N_{\mathrm{H}}>10^{23} \mathrm{~cm}^{-2}\right)$, high ionization and high velocities $(v>0.1 c)$; thus their outflow rate can be huge (several $M_{\odot} / \mathrm{yr}$ ) and they can play a key role in the co-evolution of the massive black hole and the host galaxy.

NGC 1365 is a nearby $(z=0.0055)$ Seyfert 1.8 galaxy, and it has been the target of several X-ray monitoring campaigns as it is the prototype of the so called "Changing-look" AGN (Matt et al. 2003), where the column density of the X-ray absorber changes from Compton-thin (transmission dominated state; $N_{\mathrm{H}}<10^{24} \mathrm{~cm}^{-2}$ ) to Compton-thick (reflection dominated state; $N_{\mathrm{H}}>10^{24} \mathrm{~cm}^{-2}$ ). Furthermore, NGC 1365 is also well known for the extremely rapid variability of its X-ray absorber; the monitoring campaigns and the deep Xray observations performed with Chandra, XMM-Newton and Suzaku measured a variability

of the column density (ranging from few $\times 10^{23} \mathrm{~cm}^{-2}$ to $10^{24} \mathrm{~cm}^{-2}$ ) and covering factor of the 
X-ray absorber (from $10 \%$ to $90 \%$ ) on the timescale of less than a day-weeks (Risaliti et al. 2005a, 2007, 2009a; Maiolino et al. 2010; Brenneman et al. 2013). The emerging scenario for NGC 1365 is that we are viewing the central X-ray source through an absorber made of clouds (with a column density of few $\times 10^{23} \mathrm{~cm}^{-2}$ up to $10^{24} \mathrm{~cm}^{-2}$ ) located closer in than the pc-scale torus and most likely at a distance from the central black hole of the order of $10^{16} \mathrm{~cm}$ (Risaliti et al. 2007), which is consistent with the distance of the Broad Line Region (BLR) clouds. The derived density of these clouds is also of the same order of the density of the BLR clouds (i.e. $n \sim 10^{10} \mathrm{~cm}^{-3}$ ). Another important characteristic of NGC 1365 is that when it is observed in the Compton-thin state, its X-ray spectrum shows the persistent presence of strong absorption lines due to Fexxv (both the He- $\alpha$ and He- $\beta$ ) and Fe XXVI (both the Ly- $\alpha$ and Ly- $\beta$ components); these lines are blue shifted by $v \sim 3000 \mathrm{~km} \mathrm{~s}^{-1}$ (Risaliti et al. 2005b; Brenneman et al. 2013). The intensity and velocity shift of these lines imply the presence of a highly ionised $\left(\log \xi \sim 3.51 \mathrm{erg}^{\mathrm{cm} \mathrm{s}}{ }^{-1}\right.$ ) outflowing wind with column density of a few $10^{23} \mathrm{~cm}^{-2}$ and a possible origin in an accretion disk wind. Recently it has been proposed that the long term variability of the X-ray absorber could be explained as a variable and clumpy ionized wind that is responding to the changes in the accretion rate of NGC 1365 (Connolly et al. 2014).

Despite the strong variability of the X-ray absorber and the possible variability of the primary power-law flux (Brenneman et al. 2013), the soft X-ray emission, which is normally absorbed below $2 \mathrm{keV}$, did not show any variability in either the spectral shape or in the intensity of the several emission lines (Guainazzi et al. 2009). The analysis of the $0.5 \mathrm{Ms}$ high-resolution spectrum, obtained combining all the $X M M$-Newton reflection grating spectrometer (RGS; den Herder et al. 2001) data (from the 2004 and 2007 observations), showed that the soft X-ray emission is dominated by emission lines as observed in several Seyfert 2 galaxies (Guainazzi \& Bianchi 2007). However, the emission is not purely dominated by the AGN components but requires the presence of a significant contribution from a collisionally ionised plasma. Several emission lines from He- and H-like ions (from $\mathrm{C}$ to $\mathrm{Si}$ ) as well as the L-shell transitions from FeXVII were detected; this emission line component could be thus described as an hybrid (collisionally + photoionized) gas. The collisionally ionized gas was modeled with two thermal components with temperatures of about $k T \sim 300 \mathrm{eV}$ and $k T \sim 640 \mathrm{eV}$ (Guainazzi et al. 2009), possibly associated with the strong nuclear starburst activity as suggested by the diffuse emission detected with Chandra (Wang et al. 2009).

\footnotetext{
${ }^{1}$ The ionization parameter is defined as $\xi=L_{\mathrm{ion}} / n R^{2}$, where $R$ is the distance of the ionizing source from the absorber, $n$ is the electron density and $L_{\text {ion }}$ is the ionising luminosity in the 1-1000 Rydberg range.
} 
Last year NGC 1365 was observed four more times as part of a XMM-Newton and NuSTAR (Nuclear Spectroscopic Telescope Array; Harrison et al. 2013) monitoring program; the observations were performed simultaneously to provide the optimal broadband X-ray spectra to investigate the overall X-ray emission and spectral variability. The results of the broadband analysis of the first observation (performed on July 25-27, 2012) and the determination of the possible spin parameters of the central black hole were recently published in Risaliti et al. (2013) and Walton et al. (2014). During this monitoring program NGC 1365 showed a new and even more dramatic spectral variability, which for the first time affected also the soft X-ray emission and which we consider in more detail here. While the first of these observations caught the source in its standard Compton-thin state $\left(N_{\mathrm{H}} \sim 3 \times 10^{23} \mathrm{~cm}^{-2}\right)$ during the following observations (performed in December 2012, January 2013 and February 2013) the primary power-law flux increased by a factor of 3 and more importantly during the third observation the $N_{\mathrm{H}}$ dropped to $\sim 10^{22} \mathrm{~cm}^{-2}$ (Rivers et al. in prep; Walton et al. 2014). The overall X-ray emission is now reminiscent of a weakly obscured AGN with the low energy photoelectric cut-off at about $1 \mathrm{keV}$.

We thus examined each of the XMM-Newton RGS spectra to investigate the possible signatures of this low column density absorber, our goal is to investigate its ionization state and infer its location with respect to the variable high column density absorber. In this paper we will focus on the third observation (i.e. the one performed in January 2013), where, as we will show, NGC 1365 was not only brighter but also showed for the first time a remarkable spectral variability in the RGS energy band $(0.3-1.8 \mathrm{keV})$. In order to better understand the variable component we compared this observation with the historical RGS data and with our recently obtained Chandra High-Energy Transmission Grating (HETG; Markert et al. 1994) spectrum ( 200 ks exposure time) of NGC 1365. In all these past observations the AGN is fully absorbed below $2 \mathrm{keV}$ and thus these spectra provide the template for the non variable and distant emission line component. The paper is structured as follows: in $\S 2$ we describe the XMM-Newton-RGS and Chandra observations and data reduction; in $\S 3$ we present the RGS spectral variability and the modeling of the least obscured segment of the observation. The discussion on the physical interpretation of this absorber is presented in $\S 4$. 


\section{Observations and data reduction}

\subsection{XMM-Newton}

Four $130 \mathrm{ks}$ long XMM-Newton observations of NGC 1365 were performed on July 2012, December 2012, January 2013 and February 2013. The EPIC-pn and MOS instruments were operating in full frame mode and with the medium filter applied. The results on the variability of the X-ray absorber during these new XMM-Newton-EPIC observations will be presented in a forthcoming paper (Rivers et al. in prep.). The XMM-Newton data have been processed and cleaned using the Science Analysis Software (SAS ver 12.0.1) and analyzed using standard software packages (FTOOLS ver. 6.13, XSPEC ver. 12.7; Arnaud 1996). The XMM-Newton-pn data were filtered for high background time intervals which yields net exposure time of $\sim 93 \mathrm{ksec}$. The EPIC-pn source and background spectra were extracted using a circular regions with a radius of $35^{\prime \prime}$ and of $30^{\prime \prime}$, respectively. Response matrices and ancillary response files at the source position were created using the SAS tasks arfgen and rmfgen. The source spectrum was then binned to have at least 25 counts in each energy bin. We did not use the EPIC-MOS data as they are piled-up due to the high count rate of NGC 1365 .

For the scientific analysis of this paper we concentrated on the RGS data, which have the highest spectral resolution in the soft X-ray band $(0.35-1.8 \mathrm{keV})$ with respect to the EPICCCD resolution. The RGS data have been reduced using the standard SAS task rgsproc and the most recent calibration files. High background time intervals have been filtered out applying a threshold of 0.2 counts/s on the background lightcurve extracted from a background region on the CCD-9, which is the one more affected by background flares and the closest to the optical axis. We then extracted, for each observation, the background-corrected light curves using the SAS task rgslccorr for the total RGS band adopting a binsize of $1 \mathrm{ks}$. We inspected both the light curves obtained for each RGS (considering only the first order data) as well as for the whole RGS (i.e. combining both the RGS1 and RGS2) and we found a good agreement between the RGS1 and RGS2 light curves. The inspection of these light curves shows strong variability in the RGS band only in the third observations. During this observation the two RGS collected a total of $\sim 12950$ net counts $(0.35-1.8 \mathrm{keV})$. In Fig. 1 we show the combined RGS light curve for this observation 2 .

To assess if the observed variability is caused by fast variability of the low column

\footnotetext{
${ }^{2}$ We note that the gap present in the light curve is due to the splitting of the observation into two parts.
} 
density X-ray absorber, we divided this XMM-Newton observation into 3 intervals of about $40 \mathrm{ks}$ each (vertical dashed lines in Fig:1). The intervals were chosen to have enough count statistics for a meaningful spectral analysis and according to the increasing flux trend seen in the light curve. The first interval shows only a weak increase of the RGS net counts, the second interval includes the first "flare" and steady rise, while the last one is when stronger variability is present. For each of these intervals we extracted the RGS1 and RGS2 spectra using the standard SAS task rgsproc. The two RGS collected a total of $\sim 2300, \sim 3950$ and $\sim 6700$ net counts in the first, second and third interval, respectively. The spectra were then binned at half the FWHM resolution of the instrument $(\Delta \lambda \sim 0.05 \AA)$ and the C-statistic was employed in all the fits.

\subsection{Chandra}

Chandra observed NGC 1365 twice in 2012, on April 09 and April 12, for a total of $\sim 200$ ks (OBSIDs: 13920 and 13921; see Table 1). The observations were made with the HETG instruments at the focal plane of the Advanced CCD Imaging Spectrometer (ACIS-S; Garmire et al. 2003). The HETG consists of two gratings assemblies, the High-Energy Grating (HEG; 0.7-10.0 keV) and a Medium-Energy Grating (MEG; 0.4-8 keV). The HETG data were reprocessed with the Chandra Interactive Analysis of Observations software package (CIAO version 4.43; Fruscione et al. 2006) and CALDB version 4.4.9. For each observation, events were extracted for each arm $(-1$ and +1$)$ for the first order data of each of the HETG grating (HEG and MEG). Spectral redistribution matrices ( $r m f$ files) were made with the CIAO tool $m k g r m f$ for each arm $(-1$ and +1$)$; telescope effective area files were made with the CIAO script fullgarf which drives the CIAO tool mkgarf. Spectra were extracted from these events combining the -1 and +1 orders (using response files combined with appropriate weighting); we did not subtract the background as it is negligible. For the extraction we used a narrower extraction strip than the default (20 arcsec instead of 35), the reason for this choice is to extend the HEG data above $8 \mathrm{keV}$ and also to exclude part of the extended emission from the starburst. After the inspection that the spectra extracted from the two OBSIDs are consistent, we summed them to create high quality summed first-order HEG and MEG spectra for fitting, again we created the response files combining them with the appropriate weighting. NGC 1365 was in a Compton-thick state for the whole duration of the Chandra observation. For the scientific analysis of this paper we concentrated on the MEG data, which partly overlap with the RGS spectra; we note that despite the fact that the source was in a Compton-thick state the MEG collected a total of $\sim 1700$ and $\sim 1100$

\footnotetext{
${ }^{3}$ http://cxc.harvard.edu/ciao
} 
net counts in the $0.6-7$. $\mathrm{keV}$ and $0.6-1.8 \mathrm{keV}$ energy range, respectively. For the spectral fit we then binned the MEG to 2048 channels (half of the FWHM of the spectral resolution), corresponding to $\Delta \lambda \sim 0.01 \AA$ and, as for the RGS spectral fits, we employed the C-statistic. Throughout the paper we adopted $\mathrm{H}_{0}=71 \mathrm{~km} \mathrm{~s}^{-1} \mathrm{Mpc}^{-1}, \Omega_{\Lambda}=0.73$, and $\Omega_{m}=0.27$ (Spergel et al. 2003). All the fit parameters are quoted in the rest frame of NGC 1365 and errors are at the $90 \%$ confidence level for one interesting parameter, unless otherwise stated.

\section{Spectral Fitting}

\subsection{Variability of the Soft X-ray continuum}

As NGC 1365 is known to have a highly variable X-ray absorber, we first tested if the variability shown in the RGS light curve could be explained as a variable low column density absorber. Thus we fitted the three RGS1 and RGS2 spectra with a simple baseline continuum composed of: a primary absorbed and redshifted power-law component, a soft and unabsorbed power-law component (with the same photon index $\Gamma$ ) and a thermal emission model (MEKAL model in XSPEC, Mewe et al. 1985). The latter component represents the emission due to a collisionally ionized plasma, which from the previous Chandra and RGS analysis (Wang et al. 2009; Guainazzi et al. 2009) is expected to contribute to the soft X-ray emission from NGC 1365. We included also a constant multiplicative offset between the RGS1 and RGS2, which is found to be within $\pm 4 \%$ of 1.0. In all the fits we included a Galactic column density of $N_{\mathrm{H}}=1.34 \times 10^{20} \mathrm{~cm}^{-2}$ (Kalberla et al. 2005), adopting the cross-sections and abundances of Verner et al. (1996) and Wilms et al. (2000). For all the intervals we tied the photon index of the power-law component, while we allowed the column density of the intrinsic absorber to vary. Although this fit is statistically unacceptable $(C /$ d.o.f. $=3838.1 / 2818)$ it provides a first order representation of the spectral variability. The resulting fluxed RGS spectra are shown in Fig. 2, where it is clear that the main driver of the increase of the soft X-ray flux is a decrease of the $N_{\mathrm{H}}$ of the intrinsic absorber. When parametrized by this single neutral absorber and keeping tied the normalization of the power-law components, its column density changes from $N_{\mathrm{H}}=8.3_{-1.2}^{+2.4} \times 10^{22} \mathrm{~cm}^{-2}$ to $N_{\mathrm{H}}=2.6_{-0.2}^{+0.2} \times 10^{22} \mathrm{~cm}^{-2}$ and $N_{\mathrm{H}}=0.9_{-0.1}^{+0.1} \times 10^{22} \mathrm{~cm}^{-2}$ for the first, the second and the last interval, respectively. The photon index is found to be $\Gamma=1.52 \pm 0.14$ (over the RGS band) and the temperature of the thermal component is $k T=0.34 \pm 0.02 \mathrm{keV}$, in good agreement with the value reported

in Guainazzi et al. (2009). We note that most of the remaining residuals are due to emission and absorption lines, however statistically the fit does not require the presence of a second thermal component as found in the previous work. We also tested if the difference in the spectral shape could be explained without a change of the column density of the neutral 
absorber, to this end we adopted the same baseline model but we allowed to vary only the photon index between the three intervals, the fit is worse by a $\Delta C=1329.7$ and to account for the different curvature the photon index varies from $\Gamma \sim 2.5$ to an unphysical value of $\sim-1.5$. We also allowed the normalization of the primary power-law component to vary, in this latter test the fit-statistic is better $\Delta C=68.2$ with respect to the variable absorber and the photon index varies from $\sim 1.4$ to $\sim 2.3$. However in this scenario the intensity of the primary continuum increases by a factor of 100 in the last interval, which is in disagreement with the variability seen in the broadband EPIC-pn spectra of this observation, where the primary power-law flux varies only by a factor of 2 (see $\S 3.5)$.

\subsection{The emission line spectrum}

As can be seen in Fig. 2, in the third segment we can observe the primary X-ray emission emerging above $0.8 \mathrm{keV}$ and several absorption features are present in the $1.1-1.8$ $\mathrm{keV}$ energy range, which can be associated to transitions from highly ionized $\mathrm{Ne}$ and $\mathrm{Mg}$ and suggest the presence of a ionized absorber. Before attempting any investigation of this absorber we proceeded to construct a more complex baseline model that includes the narrow emission features present in the deep RGS spectra, obtained from summing all the observations collected from 2004 and 2007 with a total exposure time of about $500 \mathrm{ks}$ for each RGS. Thus we will be able to have a correct parametrization of the absorption features (i.e $E W$, $\sigma$ and velocity shift) from which we can infer the density, ionization state and location of the low $N_{\mathrm{H}}$ absorber. For the analysis we considered also the HETG spectrum, although the exposure time is less than the combined RGS spectra the smaller extraction region provides a spectrum with less contribution from the diffuse component and it extends to higher energies (e.g. $\sim 7 \mathrm{keV}$ ). For these past observations as the $N_{\mathrm{H}}$ is $>10^{23} \mathrm{~cm}^{-2}$ we adopted as a primary continuum the scattered power-law component and the thermal emission component. Given that in the third segment we see the primary continuum, we fixed the photon-index of the power-law component to the best fit value obtained from the broadband fit $(\Gamma=2.1$; see $\S 3.4$ ) and we included successive narrow Gaussian soft X-ray emission lines assuming their width to be less than the instrumental resolution. We fit simultaneously the HETG and RGS data and, as the extraction regions are different, we allowed for a different cross normalization between the RGS and HETG spectra. Furthermore, as the smaller extraction region of the HETG spectra includes a lower contribution from the starburst emission, we allowed for different normalizations of thermal component and some of the strongest Fe XVII-XVIII emission lines in the $0.7-0.8 \mathrm{keV}$, which are most likely associated with the diffuse component. The resulting emission lines component is similar to the one presented 
in Guainazzi et al. (2009) although for some lines we have a slightly different normalization that we ascribe to the steeper photon index that was assumed in our analysis. We note that thanks to the MEG spectrum above $0.6 \mathrm{keV}$ we can deconvolve some of the emission lines into the putative AGN emission and the thermal emission components (Gofford et al. in prep).

\subsection{Absorption lines in the low column density state.}

We thus applied this baseline model for the emission component to the third interval and we allowed to vary the intrinsic column density, the normalization of the power-law component and the thermal emission component, while we kept fixed the emission line parameters. As expected, we found that the thermal emission component does not vary, being produced in the extended starburst region. Although this is a better representation of the RGS spectra, it is still a poor fit with an unacceptable statistic of C/d.o.f. $=1295.7 / 972$. Several absorption features appear to be present (see Fig. 33) at $\sim 0.65 \mathrm{keV}$ (due to O VIII), at $0.9-1.1 \mathrm{keV}$, likely due to Ne IX and Fe Xx and at $\sim 0.7 \mathrm{keV}$ (Fe XVII-Fe XVIII). Furthermore, two broader absorption features are present at $\sim 1.3 \mathrm{keV}(\mathrm{Mg} \mathrm{XI})$ and $\sim 1.5 \mathrm{keV}(\mathrm{Mg}$ XII). We note that most of the residuals are blue-ward of the corresponding emission lines, and cannot be explained with an incorrect modeling of the emission line spectrum. The only emission line that requires a different normalization with respect to the Chandra best fit is the Mg XII Ly $\alpha$. We thus added several Gaussian absorption lines; each individual line was considered to be statistically significant if the addition yielded an improvement of the fit statistic of $C>9.2$ (corresponding to $99 \%$ significance for 2 interesting parameters). We then allowed their width to be free, but keeping the width tied to that of the corresponding emission line. In particular the width of the Mg XII absorption line is tied to the Mg XII Ly $\alpha$ emission line, while for the Mg XI absorption line we tied its widths to the Mg XII Ly $\alpha$, as the MgXI is a triplet. For the Mg emission and absorption lines, we found that allowing their width to be free we get $\Delta C=7.9$ with respect to a fit with unresolved lines and $\sigma=9 \pm 3$ $\mathrm{eV}$, which corresponds to a velocity dispersion of this absorbing gas of $\sigma=1830 \pm 610 \mathrm{~km} \mathrm{~s}^{-1}$ (or FWHM $4300 \mathrm{~km} \mathrm{~s}^{-1}$ ). We also allowed the widths of the Mg XI and Mg XII to vary independently from the corresponding emission line, the fit does not improve $(\Delta C=1)$ and we found similar values of $\sigma=9_{-5}^{+8} \mathrm{eV}$ and $\sigma=9_{-5}^{+6} \mathrm{eV}$, for the Mg XI and Mg XII absorption lines respectively.

We detected 5 statistically significant absorption lines, in Table 2 we list their param- 
eters together with the most likely identification, the corresponding laboratory energy and improvement of the fit, while in Table 3 we list the parameters of the strongest emission lines as detected in this segment of the third observation. Two less significant absorption features are also present at the corresponding energies of the blue-shifted $\mathrm{O}$ VIII Ly $\alpha$ and Nex lines $(E \sim 657 \mathrm{eV}$ and $E \sim 1.029 \mathrm{keV}$, respectively) but with a $\Delta C<6$. Although we note that within the errors the measured energies of the strongest absorption lines are consistent with the rest frame energies, the energy centroids are all blue-shifted with respect to systemic with an average shift of about $\sim 1000-2000 \mathrm{~km} \mathrm{~s}^{-1}$.

The profile of the MgXII Ly $\alpha$ emission and absorption line, and to a less extent also the O viII profile, is reminiscent of a P-Cygni profile (see Fig. 4) and thus suggestive of a possible outflow. Albeit with the large uncertainties, the energy offset between the Mg XII Ly $\alpha$ emission and absorption line component is $\Delta E=14 \pm 7 \mathrm{eV}$, where the emission line is slightly redshifted; if interpreted as due to an outflowing absorber this energy shift would correspond to a velocity of this wind of $v_{\text {out }} \sim 2800 \mathrm{~km} \mathrm{~s}^{-1}$. Assuming that the emission and absorption lines are indeed produced in the same outflowing gas the similar intensity for the components of the Mg XII Ly $\alpha$ P-Cygni profile $\left(14 \mathrm{em}=6.5_{-1.5}^{+1.6} \times 10^{-5} \mathrm{ph} \mathrm{cm}^{-2} \mathrm{~s}^{-1}\right.$ and

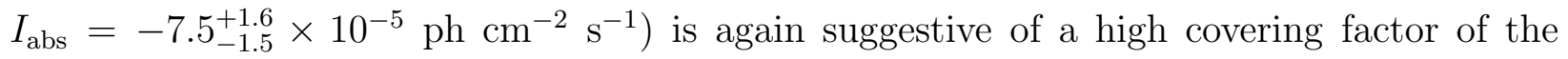
outflowing gas. However the statistics of this short RGS spectrum prevent us from a more detailed investigation of the profiles as the line parameters are subject to large uncertainties.

\subsection{Photoionization Modeling of the X-ray Absorber.}

\subsubsection{The RGS spectra}

Taking into account the fact that when NGC 1365 is observed in a Compton-thin state our line-of-sight intercepts an outflowing ionised absorber (Risaliti et al. 2005b), we attempted to model the soft X-ray spectrum replacing the absorption lines with a photoionized absorber. We adopted a model comprising a grid of photoionized absorbers generated by the XSTAR (version 2.2) photoionization code (Kallman et al. 2004); this absorber is placed in addition to the neutral absorber. The grid covers a relatively wide range in ionization (log $\xi$ ) and column density parameter space: $N_{\mathrm{H}}=1 \times 10^{20}-1 \times 10^{24} \mathrm{~cm}^{-2}$ and $\log \xi=0-6$ erg $\mathrm{cm} \mathrm{s}^{-1}$. As some of the absorption lines appear to be resolved at the RGS resolution we adopted a grid with a turbulence velocity of $1000 \mathrm{~km} \mathrm{~s}^{-1}$; this model provides an improvement of the fit of $\Delta C=22.3$ for 2 d.o.f. $(C=1268.3 / 969)$, with respect to the model

\footnotetext{
${ }^{4}$ We note that later when we adopt an xstar grid for the photoionized absorber we will derive a lower intensity for this emission line.
} 
with no absorption lines. We then allowed the absorber to be outflowing; the fit improves for an additional $\Delta C=13.1$ for 1 d.o.f. $(C=1255.2 / 968)$. However, this model cannot account for the absorption feature detected at $\sim 0.76 \mathrm{keV}$, we thus included an additional absorption line $(\Delta C=20, C=1232.2 / 965)$, the line parameters are similar to one measured with the previous model. The ionization is found to be $\log \xi=1.71_{-0.07}^{+0.09} \mathrm{erg} \mathrm{cm} \mathrm{s}^{-1}$, the column density is $N_{\mathrm{H}}=1.9_{-0.5}^{+0.8} \times 10^{22} \mathrm{~cm}^{-2}$ and the outflow velocity is $v=2100 \pm 400 \mathrm{~km} \mathrm{~s}$ ${ }^{-1}$. The additional absorption feature at $E=0.759_{-0.003}^{+0.004} \mathrm{keV}$ could suggest the presence of a second lower ionization absorber as the line is at the corresponding energy of the Unresolved Transition Array (UTA) due to $2 p \rightarrow 3 p$ transitions from low ionization M-shell iron (Fe less ionized than FeXVII). The presence of this feature generally implies the presence of an ionized absorber with $\log \xi$ of order of $0-1 \mathrm{erg} \mathrm{cm} \mathrm{s}^{-1}$. We thus tested for a double ionized absorber and despite the low exposure of these RGS spectra, we found that the $E \sim 0.76$ $\mathrm{keV}$ absorption feature can be accounted for by a low ionization absorber. The fit improves by $\Delta C=60.2(C=1175.0 / 965)$ with respect to the model with the $E \sim 0.76 \mathrm{keV}$ Gaussian absorption line. For the higher ionization component (zone 2 in Table 4) we found a higher ionization parameter $\left(\log \xi=2.1_{-0.1}^{+0.1} \mathrm{erg} \mathrm{cm} \mathrm{s}^{-1}\right.$ and $\left.N_{\mathrm{H}}=1.1_{-0.3}^{+0.4} \times 10^{22} \mathrm{~cm}^{-2}\right)$ and a slightly lower velocity $\left(v=1700 \pm 450 \mathrm{~km} \mathrm{~s}^{-1}\right)$. For the low ionization zone (zone 3 in Table 4) we found $\log \xi=0.2_{-0.1}^{+0.1} \mathrm{erg} \mathrm{cm} \mathrm{s}^{-1}, N_{\mathrm{H}}=1.1_{-0.1}^{+0.1} \times 10^{22} \mathrm{~cm}^{-2}$; its velocity is unconstrained $\left(v<2300 \mathrm{~km} \mathrm{~s}^{-1}\right)$. We note that with this low ionization absorber we do not require anymore the presence of the fully covering neutral absorber $N_{\mathrm{H}}<10^{21} \mathrm{~cm}^{-2}$, suggesting that the variable soft absorber always seen in NGC 1365 could be a low ionization zone of the disk wind.

The best fit-parameters of this model are listed in Table 4, while the RGS spectrum with the best fit model of NGC 1365 is shown in Fig. 5, It is worth noting the similarity of the outflow velocity of the zone 2 mildly ionized absorber with the velocity generally measured for the highly ionised iron $\mathrm{K}$ absorber (or zone 1 see below and Table 4, $v_{\text {out }} \sim 2000-4000$ $\mathrm{km} \mathrm{s}^{-1}$; Risaliti et al. 2005b; Brenneman et al. 2013).

\subsubsection{The EPIC-pn spectra}

Given the parameters of this ionized absorber we expect that absorption features due to He- and H-like Mg, Si and S should be present in the 1-3 keV energy range, we thus inspected the EPIC-pn spectrum extracted in the same time interval. Since we know from previous $\mathrm{X}$-ray observations that the X-ray absorber is more complex than a fully covering absorber, we included in the model a neutral partial covering absorber with no net outflow velocity. 
As for the RGS fit, we fitted the baseline model with the line emission component and we allowed to vary the intrinsic column density of all the absorbers, the normalization of the power-law component and the thermal emission component, while we kept fixed the emission line parameters; we also allowed to vary the photon index of the power-law component and the temperature of the thermal emission. The model can be parametrized as:

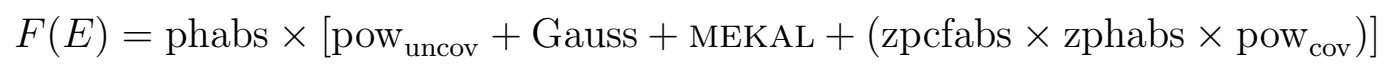

where phabs is the Galactic absorption, pow ${ }_{\text {uncov }}$ is the primary scattered power-law component, Gauss are the soft X-ray emission lines, MEKAL is the thermal emission component, zphabs is a fully covering local absorber and zpcfabs is a partial covering neutral absorber.

This model provides a poor fit to the $0.5-10 \mathrm{keV}$ spectrum $\left(\chi^{2} /\right.$ d.o.f. $\left.=1581.6 / 1264\right)$, and leaves residuals at the $\mathrm{Fe} \mathrm{K}$ emission line region due to the $\mathrm{Fe} \mathrm{K} \alpha$ emission line and the blue-shifted absorption lines. The fit is also poor below $3 \mathrm{keV}$ (see Fig. 6), confirming the prediction of the ionized absorber model that was fitted to the RGS spectra, for the presence of absorption signatures due to He- and $\mathrm{H}$-like $\mathrm{Mg}$, Si and $\mathrm{S}$. In Table 5 we report the strongest absorption lines detected in the EPIC-pn spectrum. Thus we added to the model a photoionized absorber, we also included the expected narrow Fe K $\alpha$ emission line; the fit improves by $\Delta \chi^{2}=80\left(\chi^{2} /\right.$ d.o.f. $\left.=1501.6 / 1258\right)$ and the ionization parameter is found to be similar to the value measured with the RGS spectra $\log \xi=1.4 \pm 0.2 \mathrm{erg} \mathrm{cm}$ $\mathrm{s}^{-1}$. Although the fit improves, this photoionized absorber is not able to account for the absorption due to highly ionized Fe, as the ionization is too low. Similarly if we constrain the ionization to be greater than $\log \xi>2 \mathrm{erg}^{\mathrm{cm} \mathrm{s}^{-1}}$ the fit improves by a factor of $\Delta \chi^{2}=117.9$ $\left(\log \xi=3.8 \pm 0.1 \mathrm{erg}^{\mathrm{cm} \mathrm{s}^{-1}} ; \chi^{2} /\right.$ d.o.f. $\left.=1383.7 / 1258\right)$ with respect to the low ionization one, but we are not able to account for the absorption lines detected below $3 \mathrm{keV}$.

We thus tested a model with two photoionized absorbers, allowing both their $N_{\mathrm{H}}$ and $\xi$ to vary but assuming that they have the same outflow velocity. We also included in the model an additional broad Gaussian emission line at $\sim 6 \mathrm{keV}$ to phenomenologically account for the relativistic Fe K $\alpha$ emission line (Risaliti et al. 2013) and the Fe $\mathrm{K} \beta$ emission line. For the $\mathrm{Fe} \mathrm{K} \beta$ emission line we fixed the energy centroid to $E=7.06 \mathrm{keV}$ and we tied its width to the width of the narrow Fe $\mathrm{K} \alpha$ emission line $(\sigma \sim 50 \mathrm{eV})$ and its normalisation to be $13.5 \%$ of the Fe K $\alpha$ emission line intensity (Palmeri et al. 2003). 
The model can be parametrized as:

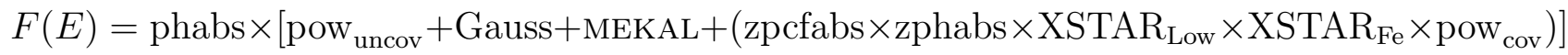

where now Gauss includes both the soft X-ray emission lines and the Fe $\mathrm{K} \alpha$ emission line complex, MEKAL is the thermal emission component, $\mathrm{XSTAR}_{\mathrm{Low}}$ and $\mathrm{XSTAR}_{\mathrm{Fe}}$ are the low and high ionization absorbers, respectively. As with the previous model zphabs is a fully covering absorber and zpcfabs is a partial covering neutral absorber.

This model improves the fit by $\Delta \chi^{2}=44.5$ for 5 d.o.f. with respect to a single ionized absorber $\left(\chi^{2} /\right.$ d.o.f. $\left.=1339.2 / 1253\right)$. The parameters of the higher ionization absorber are similar to the historically reported values $\left(\log \xi=3.7 \pm 0.1 \mathrm{erg} \mathrm{cm} \mathrm{s}{ }^{-1}\right.$ and $N_{\mathrm{H}}=1.5_{-0.5}^{+0.8} \times 10^{23} \mathrm{~cm}^{-2}$; Risaliti et al. 2005b; Brenneman et al. 2013); the outflow velocity is $v_{\text {out }}=3500_{-700}^{+1000} \mathrm{~km} \mathrm{~s}^{-1}$, while for the lower ionization zone we found $N_{\mathrm{H}}=8.4_{-2.4}^{+2.6} \times 10^{21}$ $\mathrm{cm}^{-2}$ and $\log \xi=1.5 \pm 0.2 \mathrm{erg} \mathrm{cm} \mathrm{s}^{-1}$. The ionization parameter for the lower of the two zone model for the pn data is intermediate between the values of the two RGS zones, suggesting that it is trying to fit a mix of zone 2 and zone 3 as found with the RGS data. We also allowed the two ionized absorbers to have a different outflowing velocity and we found that for the low ionization absorber we do not require any net velocity. As the higher spectral resolution of the RGS allows a more precise measurement of the outflow velocity of the low ionization component of the wind, we tested a model with the velocity of the low ionization absorber fixed to the RGS value, while we allowed the velocity of the higher ionization one to vary. Statistically the fit is equally good $\left(\chi^{2} /\right.$ d.o.f. $\left.=1330.4 / 1253\right)$ and the only parameter that varies with respect to the previous model is the outflow velocity of the high ionization absorber, which is now $v_{\text {out }}=3900_{-800}^{+800} \mathrm{~km} \mathrm{~s}^{-1}$. We thus decided to keep for the following fits the low $\xi$ velocity fixed to the RGS value.

Although the fit is already statistically good there are still some residuals in the $1.5-3$ keV energy range possibly due to emission/absorption lines from $\mathrm{Si}$ and $\mathrm{S}$, which are not accounted for by this model; thus we added several narrow Gaussian emission/absorption lines. As for the RGS analysis an individual line was considered to be statistically significant if the $\chi^{2}$ improved by a $\Delta \chi^{2}>9.2$, corresponding to $99 \%$ significance for 2 interesting parameters. The lines were assumed to be unresolved at the resolution of the EPIC-pn. Two more Gaussian absorption lines are required at $E \sim 2.5 \mathrm{keV}\left(\Delta \chi^{2}=23.3\right)$ and at $E \sim 1.83 \mathrm{keV}\left(\Delta \chi^{2}=49.9\right)$, the former line could be identified with blue-shifted He-like $\mathrm{S}\left(E_{\text {lab }}=2.461 \mathrm{keV}\right)$, while the latter is Sixiı $\left(E_{\text {lab }}=1.83 \mathrm{keV}\right)$. An identification of the first absorption line with the He-like $\mathrm{S}$ would imply an outflow velocity of $\sim 3500 \mathrm{~km} \mathrm{~s}^{-1}$. Although we note that within the error the measured energy of this absorption line is con- 
sistent with the rest frame energies, the outflow velocity is similar to the outflow velocity of the higher ionization absorber but would require a lower ionization level $(\log \xi \sim 2-2.5$ $\operatorname{erg} \mathrm{cm} \mathrm{s}^{-1}$ ), which is intermediate between the ionizations of the two-zone pn model. The presence of these two absorption features suggests either the presence of a third component of the ionized absorber or different abundances with respect to solar as assumed for the XSTAR grid. We note that in the analysis of the Suzaku observation of NGC 1365 Gofford et al. (2013) suggested the presence of a partial covering and ionized absorber with a similar ionization level ( $\log \xi \sim 2.4 \mathrm{erg} \mathrm{cm} \mathrm{s}^{-1}$; see appendix D of Gofford et al. 2013). We still require the presence of both a fully and a partial covering neutral absorber with $N_{\mathrm{H}}=(1 \pm 0.1) \times 10^{22} \mathrm{~cm}^{-2}$ and $N_{\mathrm{H} \mathrm{PC}}=1.1_{-0.3}^{+0.3} \times 10^{23} \mathrm{~cm}^{-2}$, respectively and a covering fraction for the latter of $30 \pm 10 \%$. The parameters of the ionized absorber are unaffected by the addition of these Gaussian absorption lines and consistent within the errors. We still find that the ionization and column density of the lower ionization zone $(\log \xi=1.3 \pm 0.2 \mathrm{erg}$ $\mathrm{cm} \mathrm{s}^{-1}$ and $\left.N_{\mathrm{H}}=0.7 \pm 0.2 \times 10^{22} \mathrm{~cm}^{-2}\right)$ in the pn to be intermediate between the RGS values.

We thus tested for a third component of the ionized absorber; since the RGS analysis suggests that the partial covering absorber could be a low ionization zone of the wind, we replaced the neutral partial covering absorber with a partial covering ionized absorber with no net outflowing velocity. The fit improves with respect to the two ionized absorbers and one neutral partial covering absorber $\left(\chi^{2} /\right.$ d.o.f. $\left.=1292.2 / 1253\right)$, while it is worse by a $\Delta \chi^{2}=34$ with respect to the phenomenological model with the Gaussian absorption lines. We found that the covering fraction of this third zone of the ionized absorber is $94 \pm 4 \%$. Interestingly, the column density and ionization parameter of the lower ionization zone are now $\log \xi=0.6 \pm 0.3 \mathrm{erg} \mathrm{cm} \mathrm{s}^{-1}$ and $N_{\mathrm{H}}=1.3 \pm 0.3 \times 10^{22} \mathrm{~cm}^{-2}$, which are in better agreement with the parameters of the low ionization absorber in the RGS (zone 3 in Table 4); while the parameters of the mildly ionized absorber are in good agreement with the zone 2 absorber in the RGS (see Table 4).

To summarize we have evidence for the presence of three ionized absorbers; a highly ionized and outflowing zone (zone $1 ; \log \xi \sim 3.8 \mathrm{erg} \mathrm{cm} \mathrm{s}^{-1}, v_{\text {out }}=3900_{-1000}^{+800} \mathrm{~km} \mathrm{~s}^{-1}$ ), responsible for the iron $\mathrm{K}$ absorption features and seen only in the pn data. A mildly ionized absorber seen both in the RGS and pn data (zone 2; $\log \xi \sim 2.1 \mathrm{erg} \mathrm{cm} \mathrm{s}^{-1}$ ) responsible for the $\mathrm{Mg}$, Si and $\mathrm{S}$ absorption lines, which is also outflowing $\left(v_{\text {out }}=1700_{-500}^{+400} \mathrm{~km} \mathrm{~s}^{-1}\right.$ from the RGS data); then a low ionization absorber (zone 3; $\log \xi<1 \mathrm{erg}^{\mathrm{cm} \mathrm{s}^{-1}}$ ) is responsible for the UTA, seen in the RGS, and the overall spectral curvature of the pn spectrum. Furthermore, we found that the addition of a neutral partial covering absorber improves the fit by only $\Delta \chi^{2}=11$ for 2 d.o.f. and thus is no longer strictly required. The main parameters of this 
best fit are reported in Table 4, while the residuals are shown in Fig. 6 (lower panel). The only weak residuals left are around $E \sim 0.65 \mathrm{keV}$ and $E \sim 1.6 \mathrm{keV}$, which could be ascribed to a slightly higher normalizations of the O VIII Ly $\alpha$ and $\mathrm{Mg}$ XI Ly $\beta$ emission lines in the pn spectrum with respect to the RGS best-fit. We allowed the parameters of these two emission lines to vary and we found only a marginal improvement of the fit ( $\Delta \chi^{2}=19$ for 4 d.o.f.).

\subsection{The broadband variability}

As shown by the RGS analysis the main driver of the variability detected in the soft Xray band can be explained with an uncovering of the primary X-ray source, which unveiled the presence of a mildly ionized outflowing absorber as well as the presence of emissionabsorption lines with the classical P-Cygni profile. During this observation we measured with the RGS a decrease of the column density of the partial cover absorber from $N_{\mathrm{H}} \sim 10^{23}$ $\mathrm{cm}^{-2}$ to $N_{\mathrm{H}} \sim 10^{22} \mathrm{~cm}^{-2}$ in a $130 \mathrm{ks}$ time-scale. We thus investigated the variability of the $\mathrm{X}$-ray absorber focusing on the low ionization one in the broadband EPIC-pn spectra. We divided the observation adopting the same three intervals highlighted with the RGS ligthcurves. The first inspection of these spectra suggests a variability of the curvature that can be ascribed to the well established variability of the X-ray absorber, as well as a possible variability of the intensity of the primary continuum.

We fitted simultaneously the three EPIC-pn spectra with the best-fit model found for the third segment with the 3-zones ionized absorber. As we see a clear variation of the spectral curvature both in the RGS and pn data we allowed to vary the $N_{\mathrm{H}}$ of the fully covering neutral absorber and the column density of the soft X-ray absorber (zone 3), while we kept tied its covering fraction (best fit value of $87 \pm 1 \%$, see Table 6 ). We note that the covering factor is slightly lower than the best fit value found while fitting only the third interval; this is most likely due to the assumption that it is constant during the observation. We also allowed to vary the normalization of the primary power-law component as the inspection of the spectra shows (see Fig. 7) a different flux above $8 \mathrm{keV}$. At first we kept tied the parameters of the highly and mildly ionized absorbers (zone 1 and zone 2); with this model we can test if the mildly ionized absorber could be always present but it only appears when the partial coverer absorber (zone 3) has a lower column density. Finally we allowed to vary the intensity of the Mg XII emission line, as the intensity measured with the RGS spectra is different with respect to the measurement obtained with the Chandra data, and that there is an indication of variability of the emission lines in the RGS spectra (see below and Table 7). 
This model already provides a good fit to the three spectra $\left(\chi^{2} /\right.$ d.o.f. $\left.=3909.3 / 3664\right)$, the inspection of the residuals shows that there are only some weak residuals possibly due to a change also of the ionization of the low ionized absorber or covering factor. We found evidence of variability of the continuum (primary and scattered power-law components); statistically if we constrain these intensities to be constant the fit is worse, in particular even allowing for a variability of all the three ionized absorbers $\left(N_{\mathrm{H}}\right.$ and $\left.\xi\right)$ we cannot account for the observed differences $\left(\Delta \chi^{2}\right.$ is worse by 860). We found a variability of the $N_{\mathrm{H}}$ of the fully covering neutral absorber (from $N_{\mathrm{H}}=(1.7 \pm 0.2) \times 10^{22} \mathrm{~cm}^{-2}$ to $N_{\mathrm{H}}=(0.7 \pm 0.1) \times 10^{22}$ $\mathrm{cm}^{-2}$ ) however the main driver of the spectral variability of NGC 1365 is a change in the overall column density of the low ionization zone (zone 3); which during this observation drops from $N_{\mathrm{H}}=(6.6 \pm 0.4) \times 10^{22} \mathrm{~cm}^{-2}$ to $N_{\mathrm{H}}=(0.8 \pm 0.2) \times 10^{22} \mathrm{~cm}^{-2}$.

In Table 6 we report the best fit values of this model for the three segments. We note that when fitting the three intervals keeping tied the parameters of the highly ionized wind, the outflow velocity appears to be smaller than the value measured in the last part of the observation. The short time scale variability of the highly ionized wind will be presented in Nardini et al. in prep. however, we stress that the main driver of the spectral variability is a change in the column density of the partial covering absorber (see Fig. 7).

We note that the Si and S absorption features appear to be less prominent in the first two intervals, this is similar to what has been reported for previous observations for highly ionized iron (Risaliti et al. 2005b; Brenneman et al. 2013; Gofford et al. 2013; Maiolino et al. 2010): when the primary X-ray source is more absorbed by the partial covering absorber the absorption lines are less prominent, possibly due to a lower continuum level. We found the Mg XII Ly $\alpha$ and $\operatorname{Ly} \beta$ emission lines to be weaker at the beginning of the observation when the column density of the partial coverer absorber is higher. The intensity of the Mg XII Ly $\alpha$ varied during the observation from $I<0.7 \times 10^{-6} \mathrm{ph} \mathrm{cm}^{-2} \mathrm{~s}^{-1}$ to $I=2.1 \pm 0.9 \times 10^{-5} \mathrm{ph}$ $\mathrm{cm}^{-2} \mathrm{~s}^{-1}$ and a similar variability is also seen for the $\mathrm{Mg}$ XII Ly $\beta$ (see Table 7 and Figure 8 ). The variability of the Mg XII emission line is confirmed also by the inspection of the RGS data of the three intervals; allowing its normalization to vary we found that, while in the third segment of the observation the Mg XII Ly $\alpha$ emission line is clearly detected with a flux of $I=2.5_{-1.4}^{+1.9} \times 10^{-5} \mathrm{ph} \mathrm{cm}^{-2} \mathrm{~s}^{-1}$ at the beginning of the observation we can place only an upper limit of $I<0.7 \times 10^{-5} \mathrm{ph} \mathrm{cm}^{-2} \mathrm{~s}^{-1}$. This latter value is in agreement with the intensity reported from the analysis of the $0.5 \mathrm{Ms}$ RGS data of NGC $1365\left(I=0.5 \pm 0.2 \times 10^{-5}\right.$ ph $\mathrm{cm}^{-2} \mathrm{~s}^{-1}$; Guainazzi et al. 2009). This is the opposite behaviour generally seen in the narrow emission lines that are associated with a distant emitter (i.e. the narrow line region or the obscuring torus) whose intensity is consistent with being constant. Interestingly in 
the RGS and EPIC-pn spectra of the third interval the Mg XII emission and absorption line profiles are like the classical P-Cygni profile, thus suggestive that these lines are produced in an outflowing absorber with a high covering factor, which we can associate with the mildly ionized absorber (zone 2) .

\section{The location of warm absorbers}

During this X-ray monitoring campaign of NGC 1365 we witnessed the lowest $N_{\mathrm{H}}$ and covering factor of the cold absorber and at the same time the source also increased the intrinsic flux by a factor 10 with respect to the historical values; these two facts allowed us to unveil for the first time the presence of a low ionization outflowing wind in NGC 1365. Here we briefly discuss the possible location of the warm absorbers observed in NGC 1365.

As already discussed in several works, NGC 1365 is considered the best example of a variable partial covering absorber. Prior to this monitoring program, the column density of the cold X-ray absorber was observed to vary from $N_{\mathrm{H}} \sim 10^{23}-10^{24} \mathrm{~cm}^{-2}$ (Risaliti et al. $2009 \mathrm{~b})$. The likely location of this variable cold absorber, as inferred from the time scale of the fast variability, was consistent with the BLR or the outer part of the BLR (at $R<10^{16} \mathrm{~cm}$ Brenneman et al. 2013; Maiolino et al. 2010; Risaliti et al. 2005b). Furthermore, all the Xray observations of NGC 1365 except for when the source is highly obscured, showed the presence of absorption lines due to a highly ionized wind (Brenneman et al. 2013; Risaliti et al. 2005b; Gofford et al. 2013). The detailed analysis of the spectral variability observed during the three deep Suzaku observations showed that the highly ionized component of the outflowing wind does not vary within the single observations but there is significant variability on long time-scales (Brenneman et al. 2013). In particular the ionization, the column density and the outflow velocity varied in the two years gap of the Suzaku observations between $\log \xi \sim 3-4 \mathrm{erg} \mathrm{cm} \mathrm{s}^{-1}, N_{\mathrm{H}} \sim 10^{23}-10^{24} \mathrm{~cm}^{-2}$ and $v \sim 2000-3600 \mathrm{~km} \mathrm{~s}^{-1}$. From the ionization equilibrium and the variability shown by this wind, Brenneman et al. (2013) inferred that the location of this wind is in the range of $10^{14}-3 \times 10^{15} \mathrm{~cm}$ depending on the observation, which is in agreement with the measurements reported from all the different X-ray observations of NGC 1365 and within the location of the variable cold absorber.

During the observation discussed here the highly ionized component of the absorber is characterized by a ionization state $\left(\log \xi=3.8 \pm 0.1 \mathrm{erg} \mathrm{cm} \mathrm{s}^{-1}\right)$, column density $\left(N_{\mathrm{H}}=3.5 \pm 0.9 \times 10^{23} \mathrm{~cm}^{-2}\right)$ and outflowing velocity $\left(v=2200_{-500}^{+300} \mathrm{~km} \mathrm{~s}^{-1}\right)$, which are in the range reported in literature. From these values and using the relation between the 
intrinsic continuum luminosity, the ionization and the density of the absorber $\xi=L_{\text {ion }} / n R^{2}$ (Tarter et al. 1969) we can then place a limit on the location of this absorber. Assuming then an homogenous wind and that the thickness of the absorber is less than the distance from the central BH $(\Delta R / R<1)$ this relation provides an upper limit on its distance $\left(R<L_{\text {ion }} / N_{\mathrm{H}} \xi\right)$. As during this observation the intrinsic luminosity was $L(1-1000$ Rydberg $) \sim 10^{43} \mathrm{erg} \mathrm{s}^{-1}$ (from extrapolating the best-fit over the above energy range) we derived a distance for this component of $R<10^{16} \mathrm{~cm}$; while the outflowing wind parameters are similar to the past measurements, the higher value of the maximum distance of this wind is driven by the higher luminosity with respect to the past observations. As the estimates for the mass of the super massive black hole in NGC 1365 range from $M_{\mathrm{BH}} \sim 2 \times 10^{6} \mathrm{M}_{\odot}$ to $10^{8} \mathrm{M}_{\odot}$ (Risaliti et al. 2009b), the inferred maximum distance correspond to $300-16000 r_{g}$, placing the wind within the outer part of the BLR. The minimum launch radius of this wind can be derived assuming that the outflow velocity across the line of sight is equal to the escape velocity at the observed radius; from the best fit model we found $R_{\min }>10^{4} r_{\mathrm{g}}$. As there is rather wide range for the black hole mass of NGC 1365 in the following we adopt a mean value of $10^{7} \mathrm{M}_{\odot}$.

We confirm the well known variability of the partial covering low ionisation absorber; in particular we measured a variability of the column density of about $\Delta N_{\mathrm{H}} \sim 10^{23} \mathrm{~cm}^{-2}$ during the $130 \mathrm{ks}$ exposure. Thus assuming that the obscuring clouds are moving with Keplerian velocity $\left(v_{\mathrm{k}}\right)$ a limit on the size of these clouds $\Delta R$ can be obtained using the relation $\Delta R=v_{\mathrm{k}} \Delta t$ and the duration of the observation $(\Delta t \sim 130 \mathrm{ks})$ for the elapsed time for the passage of the clouds. If the partial covering absorber is the same responsible for the Fe $\mathrm{K} \alpha$ emission line, we can then obtain an estimate of the clouds velocity from the $F W H M$ of the Fe K $\alpha$. As during the Chandra observation NGC 1365 was in a Compton-thick state, the high resolution grating data provide the best measurement of width of the Fe K $\alpha$ narrow core, which is $\sigma=30_{-11}^{+24} \mathrm{eV}$ (Gofford et al. in prep.) corresponding to a $F W H M \sim 3000$ $\mathrm{km} \mathrm{s}^{-1}$, in agreement with the value measured with the past XMM-Newton observations (Risaliti et al. 2002). Assuming a Keplerian velocity, this would correspond to a distance of these clouds of about a $10^{4} r_{g}$ and thus of the same order of the distance of the outer BLR and with a cloud size of $\Delta R<10^{14} \mathrm{~cm}$. Finally, a first order estimate of the density of these clouds can be derived from the relation $n=N_{\mathrm{H}} / \Delta R \sim 10^{9} \mathrm{~cm}^{-3}$, which is also in the typical range of the BLR clouds density. Interestingly the RGS data suggest that this variable absorber is a low ionization component of the ionized outflowing wind characterized by $\log \xi=0.63 \pm 0.03 \mathrm{erg} \mathrm{cm} \mathrm{s}^{-1}$ and a $N_{\mathrm{H}} \sim 7 \times 10^{22} \mathrm{~cm}^{-2}$ (see Table 4 and Table 6). From the estimated size of these clouds $\left(\Delta R \sim 3 \times 10^{13} \mathrm{~cm}\right)$, the density of this low ionized component of the wind and recalling that $R^{2}=L_{\text {ion }} / n \xi$ we can derive a first order estimate of the location of this variable ionized absorber of $R<10^{17} \mathrm{~cm}$. This estimate would then 
place again this low ionization absorber at a distance of the same order of the outer BLR and coincident with the location of the highly ionized outflowing wind. A possible scenario is that this zone is just the outer part of the wind and when its column density increases and or ionization decreases it could be responsible for covering-up the strong broad Mg XII emission lines and the X-ray source. The uncovering of NGC 1365, that we witnessed at the end of this observation, might be explained if we are seeing through a hole or a gap in this patchy lowly ionized absorber which is characterized by $N_{\mathrm{H}}=0.8 \pm 0.2 \times 10^{22} \mathrm{~cm}^{-2}$.

The most interesting and new result of this XMM-Newton observation of NGC 1365 has been the discovery of a mildly $\left(\log \xi=2.1 \pm 0.1 \mathrm{erg}^{\mathrm{cm} \mathrm{s}^{-1}}\right.$ see Table 4) ionized component of the outflowing wind, which is responsible for the $\mathrm{Mg}$ XI and $\mathrm{Mg}$ XII absorption features. In order to place more constraints on the location of this ionized absorber, we investigated any possible variability of its column density or ionization during the observation. However, any column density or ionization variability is strongly degenerate with the variability of the partial covering absorber. From the ionization parameter of this mildly ionization component of the wind (zone 2), its column density and using again the relation $R<L_{\text {ion }} / N_{\mathrm{H}} \xi$ we can derive only a relatively loose constraint on its distance as $R_{\max }<10^{19}$ $\mathrm{cm}$ (see Table 4). This estimate corresponds to a location outside the BLR and more typical of the putative obscuring torus; but as we discuss below the most plausible location of this absorber is closer in, at a distance smaller than the location of the variable partial covering absorber and at a similar distance of the highly ionized component (i. e. $R<10^{16} \mathrm{~cm}$ ).

As we are looking towards the innermost region through a partial covering ionized absorber (zone 3), we might be able to see the mildly ionized disk wind that imprints its features on the primary continuum, but only when the covering factor and $N_{\mathrm{H}}$ of the partial coverer are sufficiently low. Following this argument the most likely location of this mildly ionized absorber would then be within the outer part of the BLR. This scenario would naturally explain why this wind, like the highly ionized outflow, is not seen when the column density of the low ionization partial covering absorber increases, as then this part of the AGN is shielded from view. This scenario is also in agreement with the location derived from its velocity: from $v_{\text {out }}=1700 \pm 500 \mathrm{~km} \mathrm{~s}^{-1}$ we derive $R_{\min }>3 \times 10^{4} r_{\mathrm{g}}$ which for a black hole mass of $10^{7} \mathrm{M}_{\odot}$ corresponds to $R_{\min }>10^{16} \mathrm{~cm}$.

Another argument that points towards a location within the partial coverer is the detection, when the source is less obscured, of possible P-Cygni profiles for the Mg XI and Mg XII lines, which can be interpreted as arising from the zone 2 outflowing wind. As discussed in $\S 3.3$ the similarity of the strengths of the emission and absorption components of the 
P-Cygni profile indicates a covering factor close to unity of this wind, while the widths of these P-Cygni lines $\left(\sigma=9 \pm 3 \mathrm{eV}\right.$ or $\left.F W H M \sim 4300 \mathrm{~km} \mathrm{~s}^{-1}\right)$, if interpreted as velocity broadening would naturally place this wind within the putative torus and thus closer in than the classical warm absorbers $\left(R \sim 5 \times 10^{3} r_{\mathrm{g}}\right.$ or $R \sim 10^{16} \mathrm{~cm}$ for a black hole mass of $\left.M_{\mathrm{BH}} \sim 10^{7} \mathrm{M}_{\odot}\right)$. Furthermore, the inspection of the three RGS and EPIC-pn spectra provides a tentative evidence of variability, during the single XMM-Newton observation (see Table 7), of the intensity of these Mg XII emission lines. As the emission line emerges when the source is brighter and less obscured we are facing two possible scenarios: if we place this gas within the partial covering absorber (zone 3) this could be simply explained as an effect of the uncovering of the primary AGN emission. On the other hand if we place the zone 2 gas outside the partial covering absorber then the increase of the intensity of these emission lines could be explained due to a higher illuminating continuum leaking through the absorber. However, assuming that the line is responding to the illuminating continuum, the short time scale of this response implies a compact region that again would place this ionized gas within the variable X-ray absorber (zone 3); as the line is responding within the duration of the observation $(\Delta t \sim 130 \mathrm{ks}$ ) a simple estimate for the distance of the emitting gas based on the light crossing time gives a $R \sim 10^{15} \mathrm{~cm}$. Thus we conclude that the detection of this feature most likely implies a sub parsec distance for this ionized gas.

We can now estimate the mass outflow rate and energetics of this wind and compare them with the properties of the higher ionization wind. The mass outflow rate can be estimated using the expression $\dot{M}_{\text {out }}=\Omega m_{\mathrm{p}} N_{\mathrm{H}} v_{\text {out }} R$ (Krongold et al. 2007), which is appropriate for a biconical wind, where $\Omega$ is the covering factor of the wind (in terms of solid angle). For the most conservative estimate of the wind energetics we adopt the outflowing velocity as measured with the XSTAR modeling of the RGS data $\left(v_{\text {out }}=1700 \pm 500 \mathrm{~km}\right.$ $\mathrm{s}^{-1}$ ). For the distance we recall that from the ionization parameter and column density we derived an upper limit on the distance of $R_{\max }<$ few $\times 10^{19} \mathrm{~cm}$; while from the velocity broadening of the absorption lines and assuming the mean mass we can put a lower limit for this radius of about $R_{\text {min }} \sim 5 \times 10^{3} r_{\mathrm{g}}$. Given that the Mg XII emission and absorption lines are reminiscent of a P-Cygni profile, it is plausible that the mildly ionized $(\log \xi \sim 2 \mathrm{erg} \mathrm{cm}$ $\mathrm{s}^{-1}$ ) component of the wind is also responsible for the Mg XII Ly $\alpha$ emission line. Thus we estimated the global covering fraction, as a fraction of $4 \pi \mathrm{sr}$, for this absorber to produce the total observed luminosity of the Mg XII Ly $\alpha$ emission line of $L \sim 3 \times 10^{39} \mathrm{erg} \mathrm{s}^{-1}$ (from $I_{\mathrm{em}} \sim 2 \times 10^{-5}$ photons $\mathrm{cm}^{-2} \mathrm{~s}^{-1}$; see Table (7). We used the XstAR code to calculate the Mg XII line luminosity for a spherical shell of gas, covering a full $4 \pi$ sr and illuminated by the ionizing luminosity of NGC $1365\left(L_{1-1000 \text { Rydbergs }} \sim 1.5 \times 10^{43} \mathrm{erg} \mathrm{s}^{-1}\right)$, which was derived extrapolating the best fit over the $1-1000$ Rydbergs energy range. Adopting the column 
density and ionization parameter as measured for the low ionization absorber $\left(N_{\mathrm{H}} \sim 10^{22}\right.$ $\mathrm{cm}^{-2}$ and $\log \xi \sim 2 \mathrm{erg} \mathrm{cm} \mathrm{s}^{-1}$ ), we found that this absorber would produce a $\mathrm{Mg} \mathrm{XII} \mathrm{lu-}$ minosity of $6.3 \times 10^{39} \mathrm{erg} \mathrm{s}^{-1}$ for a full $4 \pi$ sr shell of gas covering the AGN. Thus, in order to reproduce the measured luminosity of $\sim 3 \times 10^{39} \mathrm{erg} \mathrm{s}^{-1}$, this absorber would require a covering fraction of about $2 \pi$.

Thus for a column density of the wind of $N_{\mathrm{H}} \sim 1 \times 10^{22} \mathrm{~cm}^{-2}$ and the above estimate of the covering fraction and distance of $R \sim 10^{16} \mathrm{~cm}$ (from equating the wind velocity to the escape velocity), we conservatively estimate $\dot{M}_{\text {out }} \sim 2 \times 10^{23} \mathrm{~g} \mathrm{~s}^{-1} \sim 3 \times 10^{-3} \mathrm{M}_{\odot} \mathrm{yr}^{-1}$ or $\dot{M}_{\text {out }} / \dot{M}_{\text {Edd }} \sim 0.01$ (assuming the accretion efficiency $\eta=0.06$ ), which means that the outflow rate is only few percent of the Eddington mass accretion rate. Assuming that the wind has reached the terminal velocity, we can also calculate the mechanical power of the wind $\dot{E}=1 / 2 \dot{M}_{\text {out }} v_{\text {out }}^{2} \sim 10^{40} \mathrm{erg} \mathrm{s}^{-1}$ and compare it with the $L_{\mathrm{Edd}} \sim 1.3 \times 10^{45} \mathrm{erg} \mathrm{s}^{-1}$, and the bolometric luminosity of NGC 1365 ( $L_{\mathrm{Bol}} \sim 10^{44} \mathrm{erg} \mathrm{s}^{-1}$, Vasudevan et al. 2010). The kinetic power of the wind is only $\sim 0.01 \%$ of the bolometric luminosity. For the highly ionized wind following the same arguments and assuming that it has the same covering factor and the averaged wind velocity of $v_{\text {out }} \sim 2300 \mathrm{~km} \mathrm{~s}^{-1}$ (see Table 6), we estimate a mass outflow rate of $\dot{M}_{\text {out }} \sim 8 \times 10^{-2} \mathrm{M}_{\odot} \mathrm{yr}^{-1}$ and a mechanical power of $\dot{E}=1 / 2 \dot{M}_{\text {out }} v_{\text {out }}^{2} \sim 10^{41}$ $\operatorname{erg~\mathrm {s}^{-1}}$, which albeit the large uncertainties is of about $\sim 0.1 \%$ of the bolometric luminosity. In both cases the wind energetics are thus below the typical values necessary for a significant feedback even considering the lower value obtained in the most recent simulation $(0.5-5 \%$ Hopkins \& Elvis 2010; Di Matteo et al. 2005; Tombesi et al. 2013). Nevertheless, the mass loss rates $\dot{M}_{\text {out }} \sim 0.3-8 \times 10^{-2} \mathrm{M}_{\odot} \mathrm{yr}^{-1}$ of the medium and highly ionized components are comparable or slightly above the mass accretion rate of NGC 1365 that can be derived from the bolometric luminosity $\left(\dot{M}_{\text {acc }} \sim 2 \times 10^{24} \mathrm{~g} \mathrm{~s}^{-1} \sim 3 \times 10^{-2} \mathrm{M}_{\odot} \mathrm{yr}^{-1}\right.$, assuming $\left.\eta=0.06\right)$. Although these estimates strongly depend on the covering factors derived from the Mg XII emission line and the XSTAR component, they suggest that both these ionized winds play

an important role in the energy and mass budgets of the active nucleus and can nevertheless have a feedback for the innermost region of the host galaxy.

\section{Conclusions}

We presented the results of a detailed analysis of one XMM-Newton observation of NGC 1365 which caught NGC 1365 in a high flux state characterized also by the lowest covering factor $(\sim 30 \%)$ and column density of the partial covering X-ray absorber ever 
recorded. Thanks to the combination of the higher flux and lower $N_{\mathrm{H}}$ we could perform a time resolved spectral analysis of the XMM-Newton-RGS data, which unveiled a variability of this low column density absorber on a time-scale as short as $40 \mathrm{ks}$. One of the main results of this observation is that the ionized disk wind present in NGC 1365 is complex and composed by at least two ionization zones, possibly three as suggested by the presence of several additional absorption lines both in the RGS and the EPIC-pn spectra. Besides the well studied highly ionized component, which is responsible for the He- and H-like Fe absorption features seen in the EPIC-pn X-ray spectra of NGC 1365, we detected a second zone with $\log \xi \sim 2 \mathrm{erg}_{\mathrm{cm} \mathrm{s}^{-1}}$ responsible for the $\mathrm{Ne}, \mathrm{Mg}$ and $\mathrm{Si}$ absorption lines. The presence of an additional absorption feature at $E \sim 0.76 \mathrm{keV}$ suggests a third lower ionization zone with $\log \xi<1 \mathrm{erg} \mathrm{cm} \mathrm{s}^{-1}$ and we propose that the variable partial covering absorber located at the BLR distance could be identified with this low ionization zone of the disk wind. We also detected two possible P-Cygni profiles, where the absorption components have widths and strengths similar to the corresponding emission components, suggestive of a wide angle outflowing wind. From the broadening of the profile and variability of the emission lines we suggest that this wind is located within the variable soft X-ray absorber.

We would like to thank the anonymous referee for his/her useful comments, which have improved this paper. This paper has made use of observations obtained with XMM-Newton an ESA science mission with instruments and contributions directly funded by ESA member states and the USA (NASA) and with the Chandra X-ray Observatory. This research has made use of software provided by the Chandra X-ray Center (CXC) in the application packages CIAO, ChIPS. J.N. Reeves acknowledges Chandra grant GO2-13123A. G. Risaliti

acknowledges the NASA grant NNX13AH71G and financial support from the Italian grant PRIN-INAF 2012.

\section{REFERENCES}

Antonucci, R. 1993, ARA\&A, 31, 473

Arnaud, K. A. 1996, Astronomical Data Analysis Software and Systems V, 101, 17

Brenneman, L. W., Risaliti, G., Elvis, M., \& Nardini, E. 2013, MNRAS, 429, 2662

Blustin, A. J., Page, M. J., Fuerst, S. V., Branduardi-Raymont, G., \& Ashton, C. E. 2005, A\&A, 431, 111

Connolly, S. D., McHardy, I. M., \& Dwelly, T. 2014, MNRAS, 440, 3503 
Crenshaw, D. M., Kraemer, S. B., \& George, I. M. 2003, ARA\&A, 41, 117

den Herder, J. W., Brinkman, A. C., Kahn, S. M., et al. 2001, A\&A, 365, L7

Di Matteo, T., Springel, V., \& Hernquist, L. 2005, Nature, 433, 604

Elvis, M., Risaliti, G., Nicastro, F., Miller, J. M., Fiore, F., \& Puccetti, S. 2004, ApJ, 615, L25

Fruscione, A., et al. 2006, Proc. SPIE, 6270

Garmire, G. P., Bautz, M. W., Ford, P. G., Nousek, J. A., \& Ricker, G. R., Jr. 2003, Proc. SPIE, 4851, 28

Gofford, J., Reeves, J. N., Tombesi, F., Braito, V., Turner, T. J., Miller, L., \& Cappi, M. 2013, MNRAS, 430, 60

Guainazzi, M., \& Bianchi, S. 2007, MNRAS, 374, 1290

Guainazzi, M., Risaliti, G., Nucita, A., et al. 2009, A\&A, 505, 589

Harrison, F. A., Craig, W. W., Christensen, F. E., et al. 2013, ApJ, 770, 103

Hopkins, P. F., \& Elvis, M. 2010, MNRAS, 401, 7

Kalberla, P. M. W., Burton, W. B., Hartmann, D., et al. 2005, A\&A, 440, 775

Kallman, T. R., Palmeri, P., Bautista, M. A., Mendoza, C., \& Krolik, J. H. 2004, ApJS, 155,675

Krongold, Y., Nicastro, F., Elvis, M., et al. 2007, ApJ, 659, 1022

Maiolino, R., Risaliti, G., Salvati, M., et al. 2010, A\&A, 517, A47

Markert, T. H., Canizares, C. R., Dewey, D., McGuirk, M., Pak, C. S., \& Schattenburg, M. L. 1994, Proc. SPIE, 2280, 168

Matt, G., Guainazzi, M., \& Maiolino, R. 2003, MNRAS, 342, 422

Mewe, R., Gronenschild, E. H. B. M., \& van den Oord, G. H. J. 1985, A\&AS, 62, 197

Palmeri, P., Mendoza, C., Kallman, T. R., Bautista, M. A., \& Meléndez, M. 2003, A\&A, 410, 359

Patrick, A. R., Reeves, J. N., Porquet, D., Markowitz, A. G., Braito, V., \& Lobban, A. P. 2012, MNRAS, 426, 2522 
Porquet, D., Reeves, J. N., O'Brien, P., \& Brinkmann, W. 2004, A\&A, 422, 85

Reynolds, C. S. 1997, MNRAS, 286, 513

Risaliti, G., Elvis, M., \& Nicastro, F. 2002, ApJ, 571, 234

Risaliti, G., Elvis, M., Fabbiano, G., Baldi, A., \& Zezas, A. 2005a, ApJ, 623, L93

Risaliti, G., Bianchi, S., Matt, G., Baldi, A., Elvis, M., Fabbiano,G., \& Zezas, A. 2005b, ApJ, 630, L129

Risaliti, G., Elvis, M., Fabbiano, G., et al. 2007, ApJ, 659, L111

Risaliti, G., et al. 2009a, MNRAS, 393, L1

Risaliti, G., et al. 2009b, ApJ, 696, 160

Risaliti, G., Harrison, F. A., Madsen, K. K., et al. 2013, Nature, 494, 449

Spergel, D. N., et al. 2003, ApJS, 148, 175

Tarter, C. B., Tucker, W. H., \& Salpeter, E. E. 1969, ApJ, 156, 943

Tombesi, F., Cappi, M., Reeves, J. N., et al. 2010a, A\&A, 521, A57

Tombesi, F., Sambruna, R. M., Reeves, J. N., Braito, V., Ballo, L., Gofford, J., Cappi, M., \& Mushotzky, R. F. 2010b, ApJ, 719, 700

Tombesi, F., Cappi, M., Reeves, J. N., Palumbo, G. G. C., Braito, V., \& Dadina, M. 2011, ApJ, 742, 44

Tombesi, F., Cappi, M., Reeves, J. N., \& Braito, V. 2012, MNRAS, 422,L1

Tombesi, F., Cappi, M., Reeves, J. N., et al. 2013, MNRAS, 430, 1102

Tombesi, F., Tazaki, F., Mushotzky, R. F., et al. 2014, arXiv:1406.7252

Vasudevan, R. V., Fabian, A. C., Gandhi, P., Winter, L. M., \& Mushotzky, R. F. 2010, MNRAS, 402, 1081

Verner, D. A., Ferland, G. J., Korista, K. T., \& Yakovlev, D. G. 1996, ApJ, 465, 487

Walton, D. J., Risaliti, G., Harrison, F. A., et al. 2014, ApJ, 788, 76

Wang, J., Fabbiano, G., Elvis, M., et al. 2009, ApJ, 694, 718 
Wilms, J., Allen, A., \& McCray, R. 2000, ApJ, 542, 914 


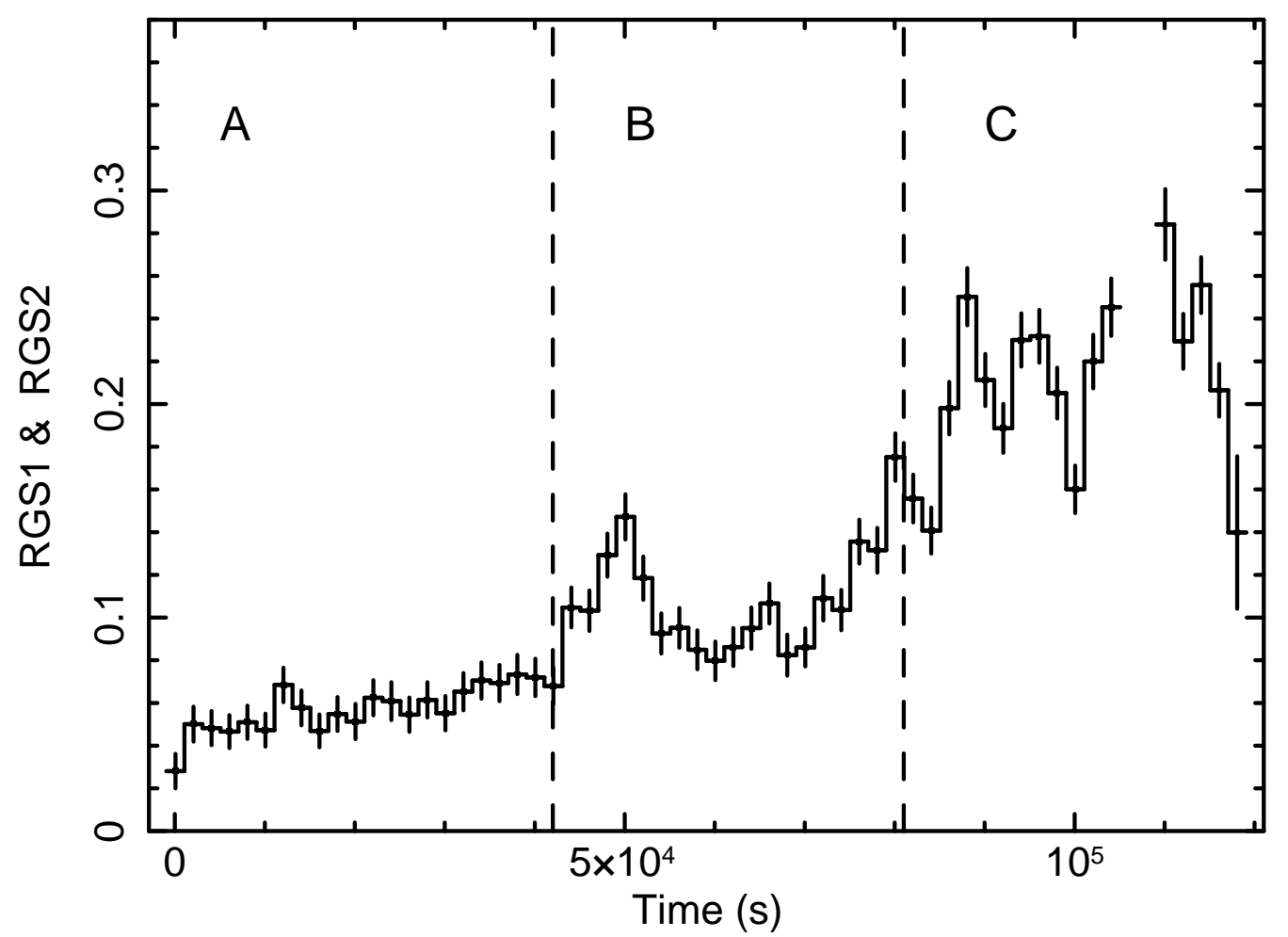

Fig. 1.- RGS net light curve binned over 2 ks showing the increase of the soft X-ray flux during the third observation. The vertical dashed lines indicate the intervals that were chosen to investigate the spectral variability in the soft X-ray band. 


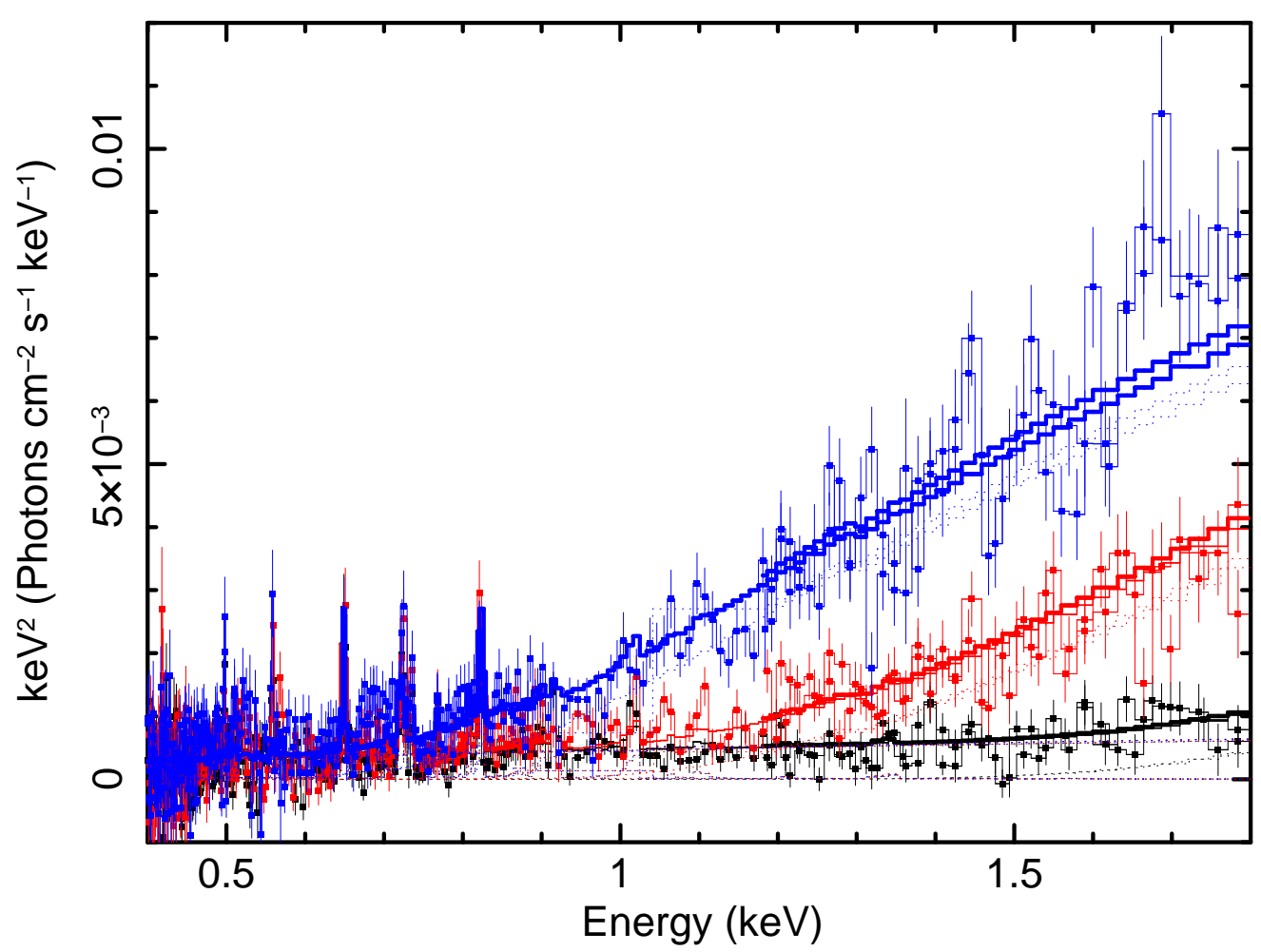

Fig. 2.- Fluxed RGS1 and RGS2 spectra obtained dividing the third observation into three intervals (black data points first $40 \mathrm{ks}$, red second interval, blue last $40 \mathrm{ks}$ ). The underlying continuum model (solid line) is composed of a primary absorbed power-law component (dotted line), a scattered component and a collisionally ionized plasma (dashed line). A clear decrease of the $N_{\mathrm{H}}$ is evident; while below $\sim 0.8 \mathrm{keV}$ the spectra are similar they clear diverge above $1 \mathrm{keV}$ due to the appearance of the primary emission. In the spectrum collected during the last $40 \mathrm{ks}$ several emission/absorption features are present. In particular in the $1.1-1.7 \mathrm{keV}$ the spectrum shows a clear imprint of a possible ionized absorber. 

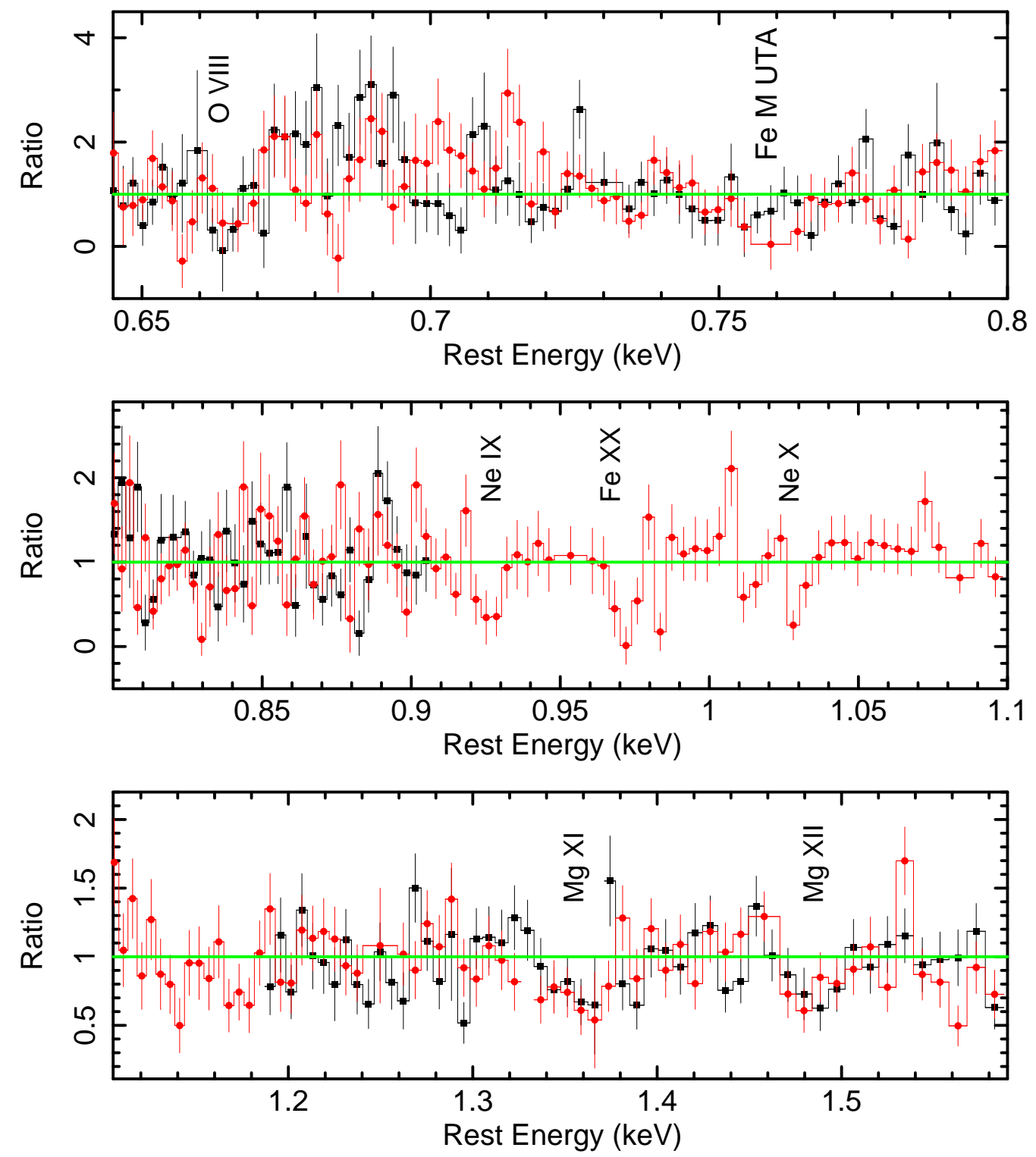

Fig. 3.- Residuals in the RGS against the baseline model that includes the emission lines detected in the 2004-2007 RGS spectra. Several absorption lines can be clearly seen. The upper panel shows the Fe M-shell band, the middle panel shows the absorption due the Ne IX and Nex; the lower panels shows absorption Mg XI and Mg XII. 

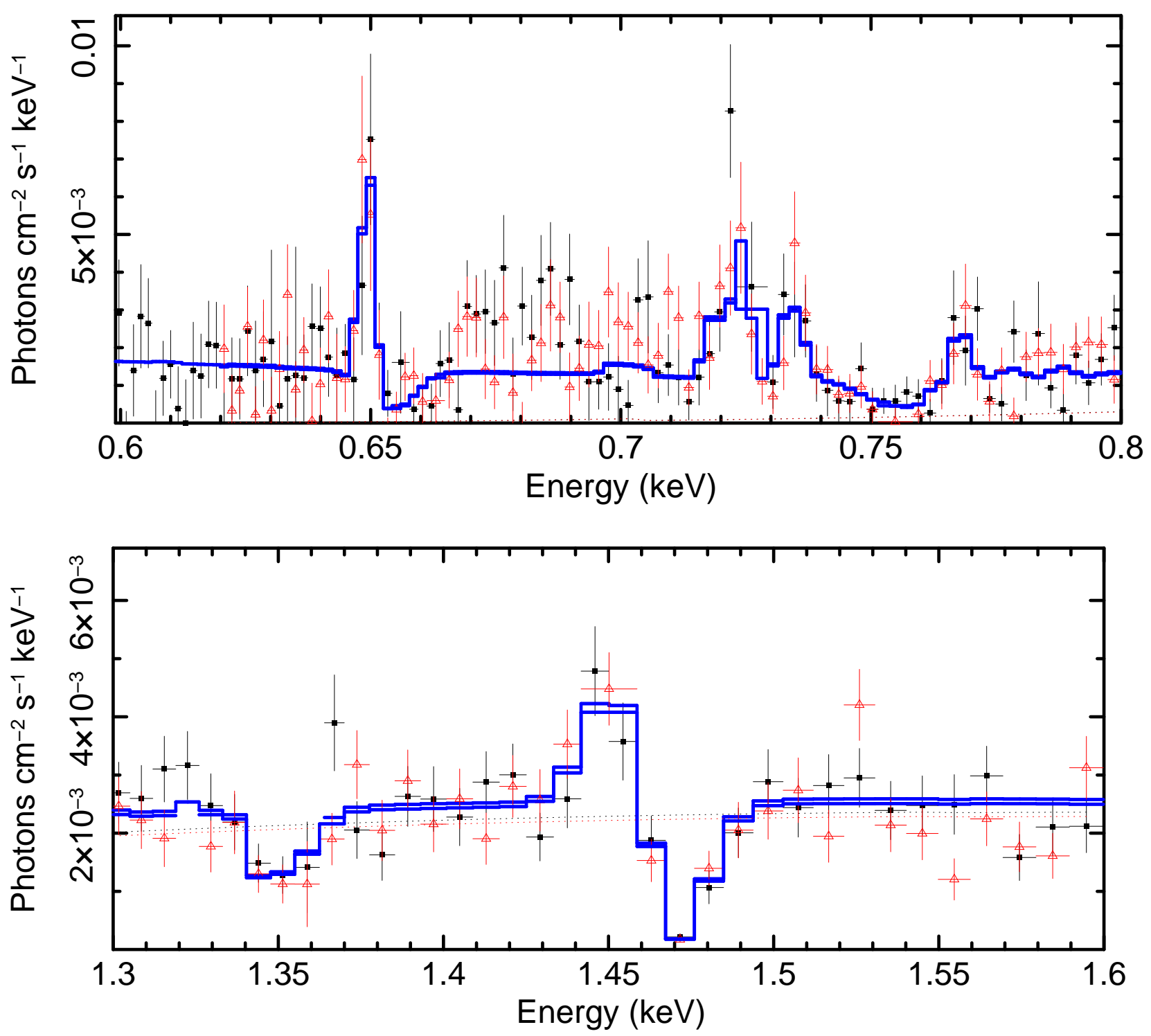

Fig. 4.- RGS unfolded spectrum showing some of the absorption lines. The upper panel shows a zoom into the O VIII and Fe M-shell region. The lower panel shows the Mg XI and Mg XII classical P-Cygni profiles, composed of an emission and blue-shifted absorption line. The profiles were fit with a double Gaussian model. 


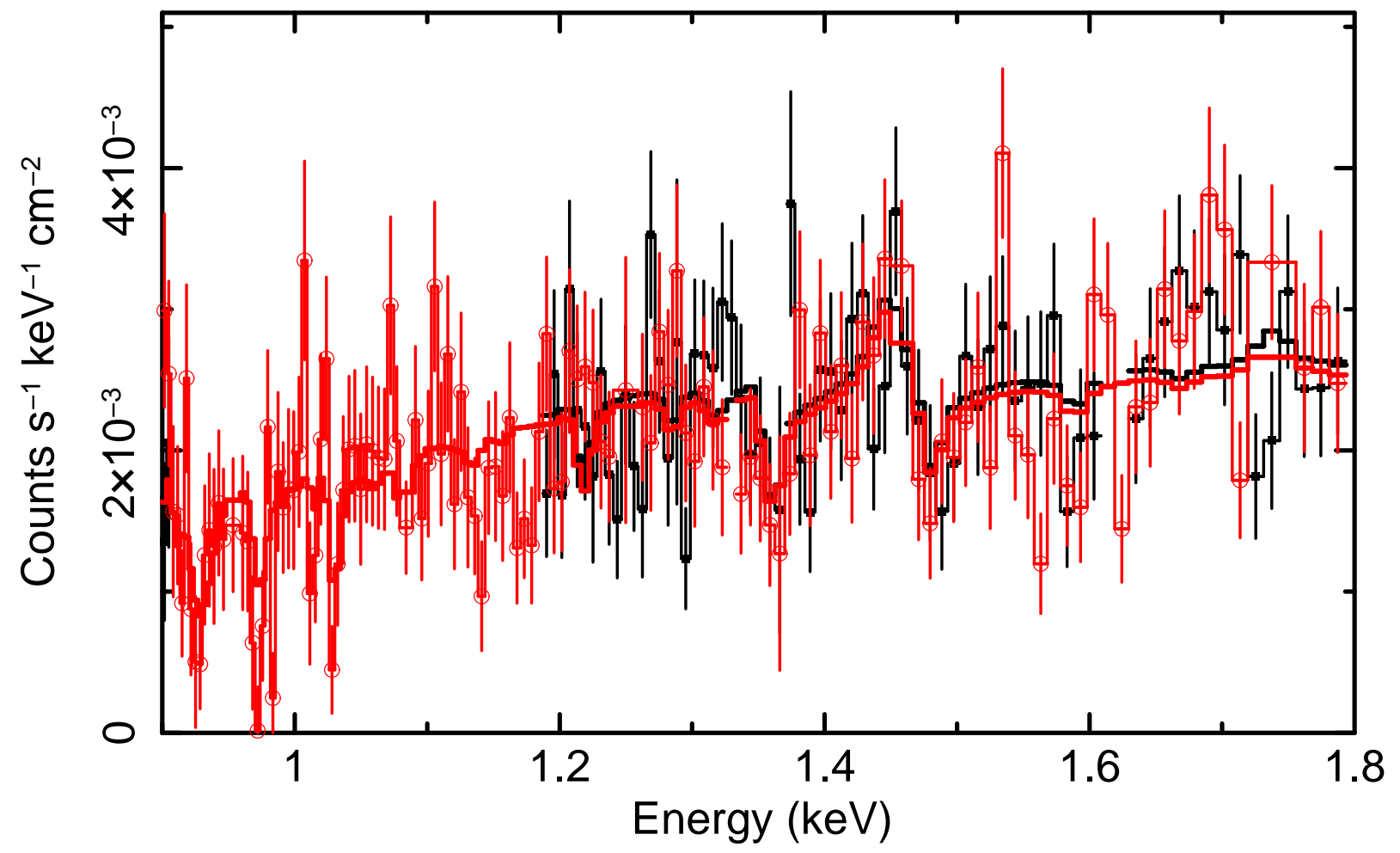

Fig. 5.- Zoom into the 0.9-1.8 keV RGS spectra (RGS1: black data filled points, RGS2: red open data points), with the XSTAR best fit model. The model for the X-ray absorber is composed of two ionized absorbers with a similar column density of $N_{\mathrm{H}} \sim 10^{22} \mathrm{~cm}^{-2}(\log$ $\xi \sim 2 \mathrm{erg}_{\mathrm{cm} \mathrm{s}}^{-1}$ and $\log \xi \sim 0.2 \mathrm{erg}^{\mathrm{cm} \mathrm{s}^{-1}}$; see Table 4). 


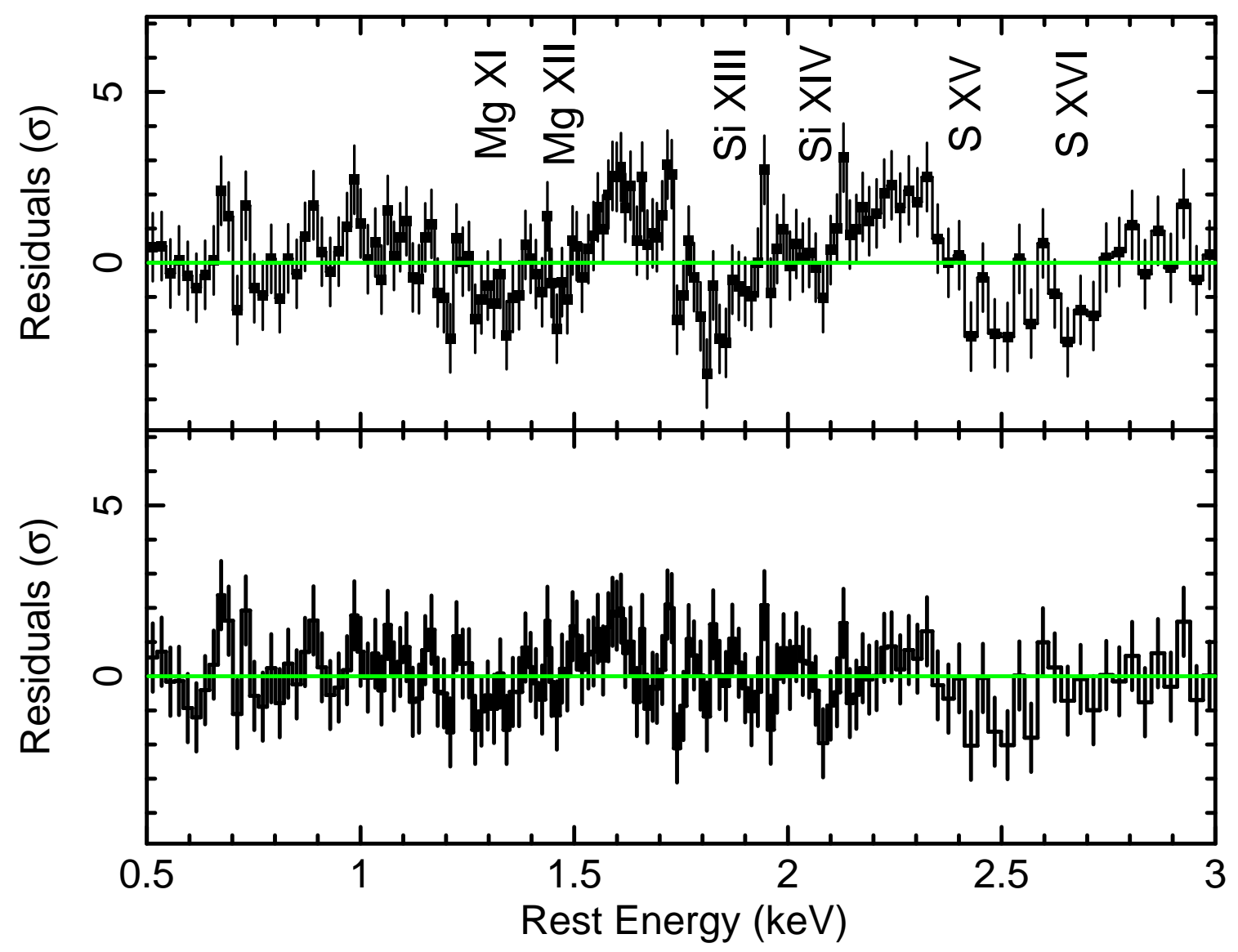

Fig. 6.- Upper panel: Zoom into the $0.5-3 \mathrm{keV}$ EPIC-pn residuals (in $\sigma$ ) against the emission line model. The absorption lines detected in the RGS energy range are also clearly present in the EPIC-pn spectrum, furthermore some higher energy absorption features are present. The strongest absorption features are labelled. Lower panel: Zoom into the 0.5$3 \mathrm{keV}$ EPIC-pn residuals (in $\sigma$ ) against the best fit model which includes three ionized absorbers. 


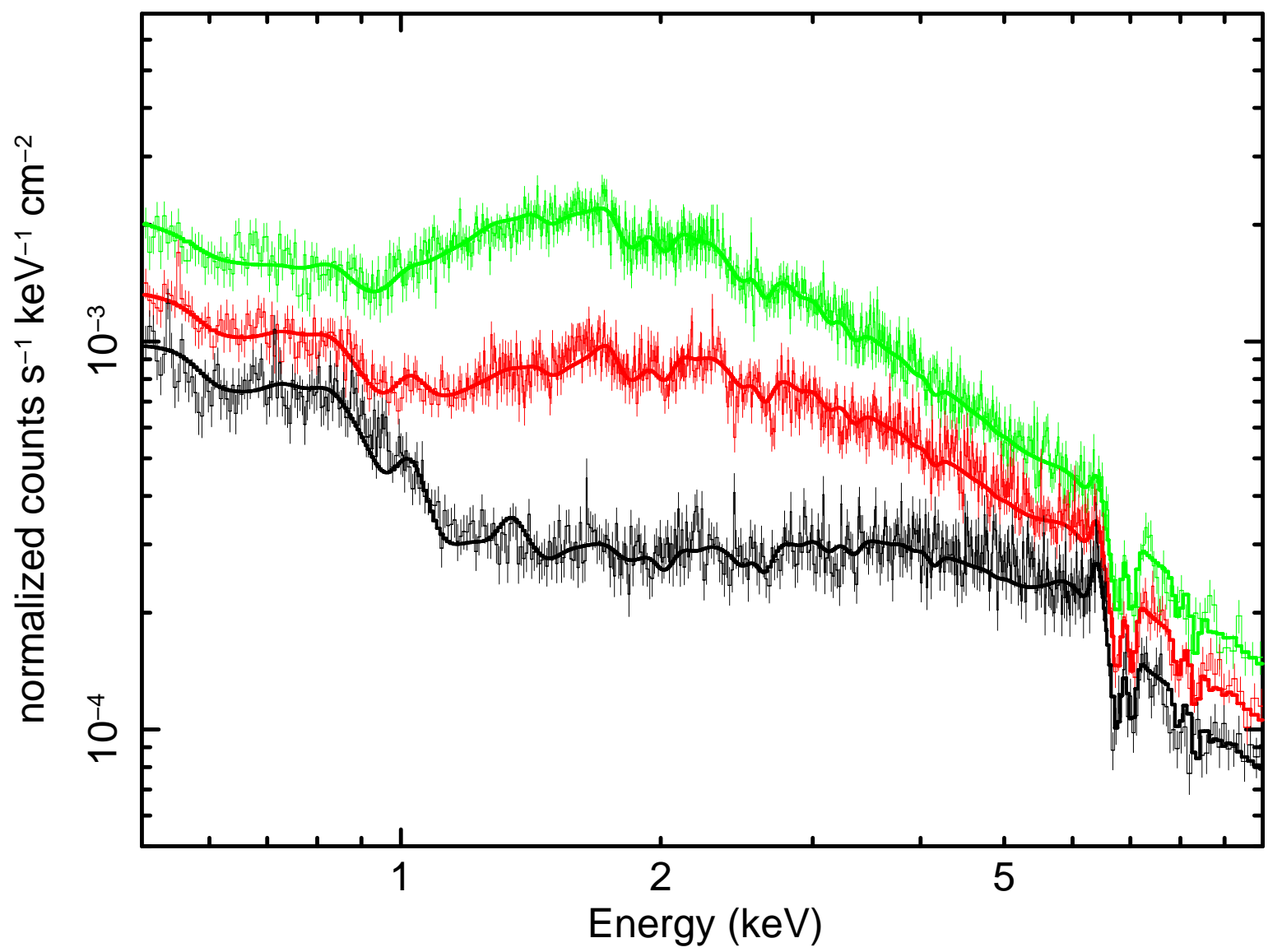

Fig. 7.- EPIC-pn spectra of the three intervals obtained dividing the observation with the same GTI adopted for the RGS analysis (black data points first $40 \mathrm{ks}$, red second interval, green last $40 \mathrm{ks}$ ). The best-fit model for the X-ray absorber is composed of: a fully covering neutral absorber, two ionized and outflowing absorbers and a ionized partial covering absorber. A decrease of the amount of absorption of the low ionization one is evident with a change in $N_{\mathrm{H}}$ from $\sim 7 \times 10^{22} \mathrm{~cm}^{-2}$ to $N_{\mathrm{H}} \sim 8 \times 10^{21} \mathrm{~cm}^{-2}$. 

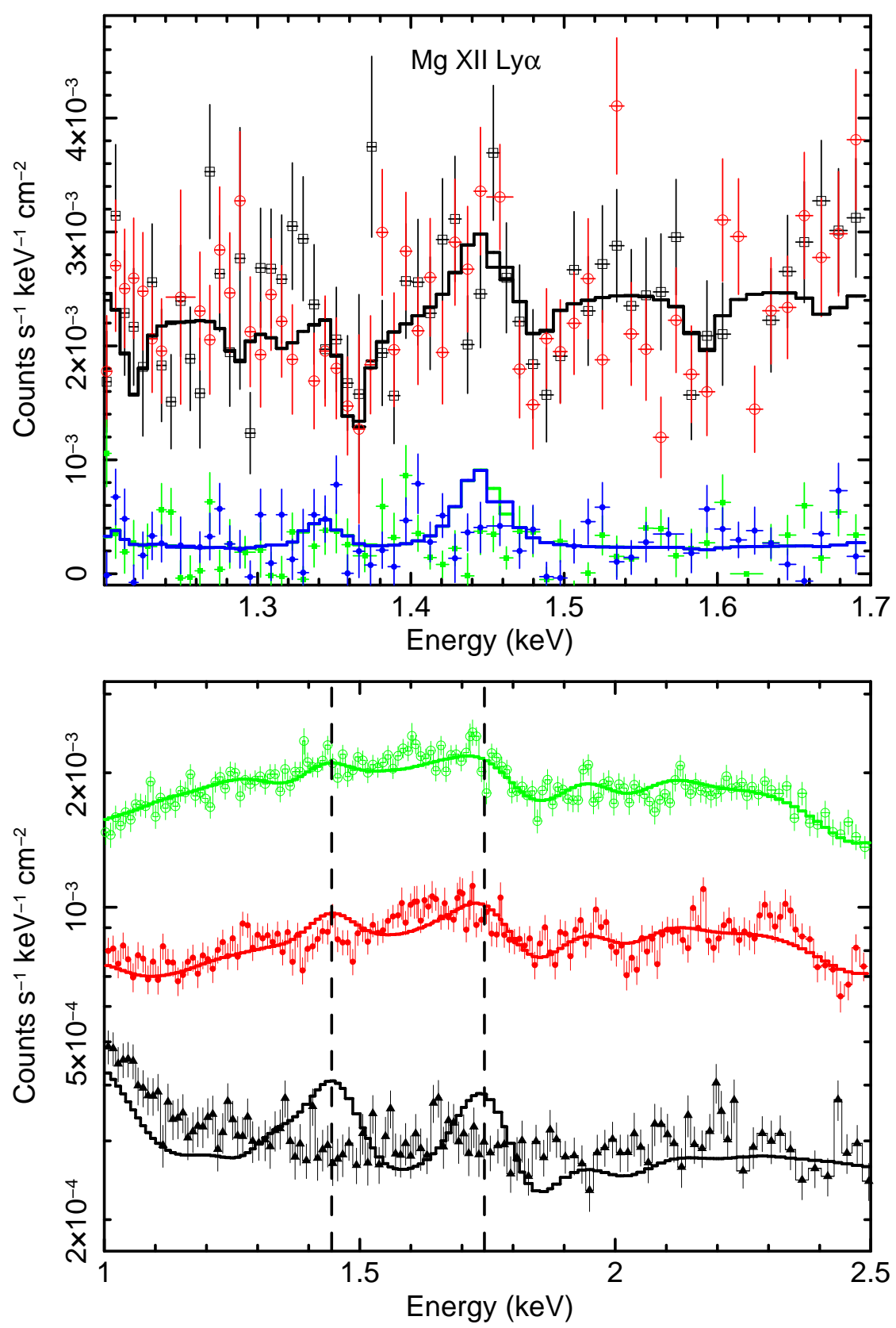

Fig. 8. - Zoom into the Mg XII energy range. To highlight the variability of the emission lines (see Table 7) their intensities were fixed to the best fit found for the last part of the observation. Upper panel: RGS spectra of the first (filled circles and squares data points; blue and green in the electronic version) and last intervals (open circles and squares data points; black and red in the electronic version). Lower panel: EPIC-pn spectra for the three intervals (filled triangles interval A, filled squares interval B and open circles interval C). The vertical dashed lines indicate the $\operatorname{Mg}$ XII Ly $\alpha(E \sim 1.45 \mathrm{keV})$ and the Mg XII Ly $\beta$ $(E \sim 1.74 \mathrm{keV})$ emission lines, respectively. 
Table 1. Log of the observations and exposure times.

\begin{tabular}{ccccc}
\hline \hline Mission & Instrument & $\mathrm{T}_{\text {(total) }}(\mathrm{ks})$ & $\mathrm{T}_{(\mathrm{net})}(\mathrm{ks})$ & DATE \\
\hline XMM-Newton & PN & 132.2 & 92.7 & $2013-01-23$ \\
XMM-Newton & RGS1 & 133.7 & 113.2 & $2013-01-23$ \\
XMM-Newton & RGS2 & 133.7 & 113.3 & $2013-01-23$ \\
Chandra & ACIS-S HETG & 90 & - & $2012-04-09$ \\
Chandra & ACIS-S HETG & 110 & - & $2012-04-12$ \\
\hline
\end{tabular}

Note. - For XMM-Newton the net exposure times are after filtering for high-background time intervals.

Table 2. XMM-Newton RGS1 and RGS2: best fit absorption lines required in the third interval. The statistic for the model with no lines is $C /$ d.o.f. $=1290.6 / 971$

\begin{tabular}{cccccc}
\hline \hline $\begin{array}{c}\text { Rest Energy } \\
(\mathrm{eV})\end{array}$ & $\begin{array}{c}\text { Intensity } \\
\left(10^{-5} \mathrm{ph} \mathrm{cm}^{-2} \mathrm{~s}^{-1}\right)\end{array}$ & $\begin{array}{c}\sigma^{\mathrm{b}} \\
(\mathrm{eV})\end{array}$ & ID $^{\mathrm{b}}$ & $\begin{array}{c}\text { Atomic Energy }^{\mathrm{d}} \\
(\mathrm{eV})\end{array}$ & $\Delta C^{\mathrm{e}}$ \\
& & & & & \\
\hline $760.4_{-5.4}^{+5.7}$ & $-1.57_{-0.70}^{+0.76}$ & $7 \pm 5$ & Fe XVII -XVIII & $\ldots$ & 10.8 \\
$925.7_{-2.3}^{+2.8}$ & $-1.39_{-0.79}^{+0.59}$ & $<4$ & Ne IX & 922.0 & 11.4 \\
$971.6_{-1.9}^{+1.6}$ & $-1.95_{-0.46}^{+0.73}$ & $<4$ & Fe XX2p $-3 d$ & 967.3 & 16.9 \\
$1355.4_{-7.6}^{+7.9}$ & $-3.46_{-1.21}^{+1.26}$ & $9 \pm 3$ & Mg XI & 1352.2 & 13.5 \\
$1476.5_{-4.9}^{+3.9}$ & $-7.46_{-1.47}^{+1.55}$ & $9^{\mathrm{f}}$ & Mg XII & 1472.6 & 17.5 \\
\hline
\end{tabular}

Note. - The $\Gamma$ of the soft power-law is tied to the hard power-law component and is fixed to the best fit value for the EPIC-pn spectrum.

a Measured line energy in the NGC 1365 rest frame.

${ }^{\mathrm{b}} 1 \sigma$ width of the absorption line in $\mathrm{eV}$.

c Possible identification.

d Known atomic energy of the most likely identification of the line in $\mathrm{eV}$.

e Improvement in C-statistic upon adding the line.

f Tied to the Mg XI line width. 
Table 3. XMM-Newton RGS1 and RGS2: best fit parameters of the strongest emission lines required in the third interval.

\begin{tabular}{ccccc}
\hline \hline $\begin{array}{c}\text { Rest Energy } \\
(\mathrm{eV})\end{array}$ & $\begin{array}{c}\text { Intensity } \\
\left(10^{-5} \mathrm{ph} \mathrm{cm}^{-2} \mathrm{~s}^{-1}\right)\end{array}$ & $\mathrm{ID}^{\mathrm{b}}$ & $\begin{array}{c}\text { Atomic Energy } \\
(\mathrm{eV})\end{array}$ & $\Delta C^{\mathrm{e}}$ \\
& & & & \\
\hline $499.9_{-0.5}^{+0.5}$ & $2.56_{-1.38}^{+0.68}$ & N VII & 500.4 & 16.9 \\
$560.5_{-0.8}^{+0.8}$ & $3.12_{-1.39}^{+1.43}$ & O VII & 561.0 & 13.6 \\
$653.2_{-1.5}^{+1.7}$ & $0.98_{-0.69}^{+0.76}$ & O VIII & 653.7 & 9.2 \\
$1462.0_{-5.8}^{+4.9}$ & $6.54_{-1.51}^{+1.61}$ & Mg XII & 1472.6 & 11.3 \\
\hline
\end{tabular}

Note. - ${ }^{a}$ Measured line energy in the NGC 1365 rest frame.

b Possible identification.

${ }^{c}$ Known atomic energy of the most likely identification of the line in $\mathrm{eV}$. 
Table 4. XMM-Newton RGS and EPIC-pn best fit parameters for the ionized absorber model. The statistics for the model are C/d.o.f. $=1175.0 / 965$ and $\chi^{2} /$ d.o.f. $=1292.2 / 1253$ for the RGS and the EPIC-pn spectra respectively.

\begin{tabular}{|c|c|c|c|}
\hline Component & Parameter & RGS & EPIC-pn \\
\hline \multirow[t]{3}{*}{ Power-law } & $\Gamma$ & $2.1^{\mathrm{f}}$ & $2.05_{-0.06}^{+0.04}$ \\
\hline & $A_{\text {scattered }}^{\mathrm{a}}$ & $2.4_{-0.9}^{+0.8}$ & $3.1_{-1.6}^{+1.3}$ \\
\hline & $A_{\text {primary }}^{\mathrm{b}}$ & $2.1_{-0.2}^{+0.2}$ & $1.5_{-0.2}^{+0.1}$ \\
\hline ionised absorber & $N_{\mathrm{H}}^{\mathrm{c}}$ & - & $21.5_{-7.8}^{+11.8}$ \\
\hline \multirow[t]{2}{*}{ zone 1} & $\log \xi^{\mathrm{d}}$ & - & $3.77_{-0.09}^{+0.12}$ \\
\hline & $v_{\text {out }}^{\mathrm{e}}$ & - & $3900_{-1000}^{+800}$ \\
\hline ionised absorber & $N_{\mathrm{H}}^{\mathrm{c}}$ & $1.1_{-0.3}^{+0.4}$ & $1.0_{-0.2}^{+0.3}$ \\
\hline \multirow[t]{2}{*}{ zone 2} & $\log \xi^{\mathrm{d}}$ & $2.15_{-0.10}^{+0.12}$ & $2.11_{-0.08}^{+0.16}$ \\
\hline & $v_{\text {out }}^{\mathrm{e}}$ & $1700_{-500}^{+400}$ & $=\mathrm{RGS}$ \\
\hline Fully covering absorber & $N_{\mathrm{H}}^{\mathrm{c}}$ & $<0.1$ & $0.3_{-0.1}^{+0.3}$ \\
\hline $\begin{array}{l}\text { Partial covering } \\
\text { ionised absorber } \\
\text { zone } 3\end{array}$ & $\begin{array}{c}N_{\mathrm{H}}^{\mathrm{c}} \\
\log \xi^{\mathrm{d}} \\
\mathrm{CF}\end{array}$ & $\begin{array}{c}1.1_{-0.1}^{+0.1} \\
0.17_{-0.09}^{+0.08} \\
1^{g}\end{array}$ & $\begin{array}{c}1.3_{-0.3}^{+0.2} \\
0.6_{-0.3}^{+0.2} \\
0.94 \pm 0.04\end{array}$ \\
\hline
\end{tabular}

Note. -

a Normalization in units of $10^{-4}$ photons $\mathrm{cm}^{-2} \mathrm{~s}^{-1}$.

b Normalization in units of $10^{-2}$ photons $\mathrm{cm}^{-2} \mathrm{~s}^{-1}$.

c $N_{\mathrm{H}}$ in units of $10^{22} \mathrm{~cm}^{-2}$.

${ }^{\mathrm{d}}$ Log of the ionization parameter, in units of erg $\mathrm{cm} \mathrm{s}^{-1}$.

e Outflow velocity in $\mathrm{km} \mathrm{s}^{-1}$.

${ }^{f}$ Denotes the parameter is fixed.

g As the RGS has a limited bandpass we assumed a covering fraction of unity for the zone 3 absorber. 
Table 5. XMM-Newton EPIC-pn soft X-ray absorption lines.

\begin{tabular}{ccccc}
\hline \hline $\begin{array}{c}\text { Rest Energy }^{\mathrm{a}} \\
(\mathrm{keV})\end{array}$ & $\begin{array}{c}\text { Intensity } \\
\left(10^{-5} \mathrm{ph} \mathrm{cm}^{-2} \mathrm{~s}^{-1}\right)\end{array}$ & ID $^{\mathrm{b}}$ & $\begin{array}{c}\text { Atomic Energy } \\
(\mathrm{keV})\end{array}$ & $\left(\Delta \chi^{2}\right)^{\mathrm{d}}$ \\
& & & & \\
\hline $0.77_{-0.03}^{+0.03}$ & $-3.5_{-1.5}^{+1.6}$ & Fe XVII -XVIII & $\ldots$ & 14.2 \\
$1.33_{-0.02}^{+0.03}$ & $-3.0_{-0.9}^{+0.9}$ & Mg XI & 1.33 & 18.0 \\
$1.47_{-0.02}^{+0.02}$ & $-3.1_{-1.0}^{+0.9}$ & Mg XII & 1.47 & 16.9 \\
$1.84_{-0.02}^{+0.02}$ & $-4.0_{-1.0}^{+1.1}$ & Si XIII & 1.84 & 37.4 \\
$2.49_{-0.03}^{+0.04}$ & $-2.2_{-1.0}^{+0.9}$ & S XV & 2.46 & 16.7 \\
$6.77_{-0.02}^{+0.03}$ & $-2.2_{-0.4}^{+0.4}$ & Fe XXV & 6.63 & 66.4 \\
$7.05_{-0.02}^{+0.03}$ & $-2.1_{-0.4}^{+0.5}$ & Fe XXVI & 6.97 & 60.7 \\
\hline
\end{tabular}

Note. - ${ }^{a}$ Measured line energy in the NGC 1365 rest frame.

b Possible identification.

${ }^{c}$ Known atomic energy of the most likely identification of the line in $\mathrm{eV}$.

d $\left(\Delta \chi^{2}\right)$ with respect to a fit without the two ionized absorbers, which provides $\chi^{2} /$ d.o. $f=$ $1500.7 / 1258$ 
Table 6. XMM-Newton-EPIC-pn best fit parameters for the 3 ionized absorbers model. The statistics for the model is $\chi^{2} /$ d.o.f. $=3909.3 / 3664$.

\begin{tabular}{ccccc}
\hline \hline Component & Parameter & $\mathrm{A}$ & $\mathrm{B}$ & $\mathrm{C}$ \\
& & & & \\
\hline Power-law & $A_{\text {Scattered }}^{\mathrm{a}}$ & $2.1_{-0.1}^{+0.1}$ & $3.1_{-0.1}^{+0.1}$ & $5.1_{-0.2}^{+0.2}$ \\
& $A_{\text {primary }}^{\mathrm{b}}$ & $0.62_{-0.04}^{+0.04}$ & $0.84_{-0.02}^{+0.04}$ & $1.22_{-0.06}^{+0.05}$ \\
Fully covering absorber & $N_{\mathrm{H}}^{\mathrm{c}}$ & $2.0_{-0.1}^{+0.2}$ & $0.96_{-0.07}^{+0.06}$ & $0.84_{-0.05}^{+0.09}$ \\
& & & & \\
ionised absorber & $N_{\mathrm{H}}^{\mathrm{c}}$ & $35.3_{-8.9}^{+8.1}$ & $35.3^{t}$ & $35.3^{t}$ \\
zone 1 & $\log \xi^{\mathrm{d}}$ & $3.81_{-0.06}^{+0.05}$ & $3.81^{t}$ & $3.81^{t}$ \\
& $v_{\mathrm{out}}^{\mathrm{e}}$ & $2300_{-400}^{+500}$ & $2300^{t}$ & $2300^{t}$ \\
ionised absorber & $N_{\mathrm{H}}^{\mathrm{c}}$ & $1.7_{-0.3}^{+0.3}$ & $1.7^{t}$ & 1.7 \\
zone 2 & $\log \xi^{\mathrm{d}}$ & $2.3_{-0.1}^{+0.1}$ & $2.3^{t}$ & $2.3^{t}$ \\
& & & & \\
Partial covering & & & & \\
ionised absorber & $N_{\mathrm{H}}^{\mathrm{c}}$ & $7.0_{-0.5}^{+0.4}$ & $2.4_{-0.1}^{+0.1}$ & $0.8_{-0.2}^{+0.1}$ \\
zone 3 & $\log \xi^{\mathrm{d}}$ & $0.63_{-0.02}^{+0.03}$ & $0.63^{t}$ & $0.63^{t}$ \\
& $\mathrm{CF}$ & $0.87 \pm 0.01$ & $0.87^{t}$ & $0.87^{t}$ \\
\hline
\end{tabular}

Note. -

a Normalization, in units of $10^{-4}$ photons $\mathrm{cm}^{-2} \mathrm{~s}^{-1}$.

b Normalization, in units of $10^{-2}$ photons $\mathrm{cm}^{-2} \mathrm{~s}^{-1}$.

c $N_{\mathrm{H}}$ in units of $10^{22} \mathrm{~cm}^{-2}$.

${ }^{\mathrm{d}} \log$ of the ionization parameter, in units of $\mathrm{erg} \mathrm{cm} \mathrm{s}^{-1}$.

e Outflow velocity in $\mathrm{km} \mathrm{s}^{-1}$.

g Fully covering absorber.

${ }^{\mathrm{t}}$ Denotes the parameter is tied. 
Table 7. Intensities of the Mg XII emission lines

\begin{tabular}{cccc}
\hline \hline & RGS & \multicolumn{2}{c}{ EPIC-pn } \\
\hline Interval & Mg XII Ly $\alpha$ & Mg XII Ly $\alpha$ & Mg XII Ly $\beta$ \\
& $\left(10^{-5} \mathrm{ph} \mathrm{cm}^{-2} \mathrm{~s}^{-1}\right)$ & $\left(10^{-5} \mathrm{ph} \mathrm{cm}^{-2} \mathrm{~s}^{-1}\right)$ & $\left(10^{-5} \mathrm{ph} \mathrm{cm}^{-2} \mathrm{~s}^{-1}\right)$ \\
& & & \\
\hline INT A & $<0.7$ & $<0.07$ & $<0.3$ \\
INT B & $<1.3$ & $<0.3$ & $<0.9$ \\
INT C & $2.5_{-1.4}^{+1.9}$ & $2.1_{-0.9}^{+0.9}$ & $1.9_{-1.3}^{+1.6}$ \\
\hline
\end{tabular}

